\title{
Type 2 diabetes risk gene Dusp8 regulates hypothalamic Jnk signaling and insulin sensitivity
}

\author{
Sonja C. Schriever, ${ }^{1,2,3}$ Dhiraj G. Kabra, ${ }^{1,2,3,4}$ Katrin Pfuhlmann, ${ }^{1,2,3,5}$ Peter Baumann, ${ }^{1,2,3,6}$ Emily V. Baumgart, ${ }^{1,2,3}$ Joachim Nagler, ${ }^{7}$ \\ Fabian Seebacher, ${ }^{2,3,5}$ Luke Harrison, ${ }^{1,2,3,5}$ Martin Irmler, ${ }^{8}$ Stephanie Kullmann, ${ }^{3,9}$ Felipe Corrêa-da-Silva, ${ }^{10,11}$ Florian Giesert, ${ }^{12,13}$ \\ Ruchi Jain, ${ }^{2,3,14}$ Hannah Schug, ${ }^{15}$ Julien Castel, ${ }^{16}$ Sarah Martinez, ${ }^{16}$ Moya Wu, ${ }^{8}$ Hans-Ulrich Häring, ${ }^{3,9,17}$ Martin Hrabe de Angelis, ${ }^{3,8,18}$ \\ Johannes Beckers, ${ }^{3,8,18}$ Timo D. Müller, ${ }^{2,3,19}$ Kerstin Stemmer, ${ }^{2,3}$ Wolfgang Wurst, ${ }^{12,13,20,21}$ Jan Rozman, ${ }^{3,8,22}$ Ruben Nogueiras, ${ }^{23}$ \\ Meri De Angelis, ${ }^{7}$ Jeffery D. Molkentin, ${ }^{24,25}$ Natalie Krahmer, ${ }^{2,3}$ Chun-Xia Yi, ${ }^{10,11}$ Mathias V. Schmidt, ${ }^{26}$ Serge Luquet, ${ }^{16}$ \\ Martin Heni, ${ }^{3,9,17,27}$ Matthias H. Tschöp, ${ }^{2,3,5}$ and Paul T. Pfluger ${ }^{1,2,3,6}$
}

'Research Unit NeuroBiology of Diabetes and Institute for Diabetes and Obesity, Helmholtz Zentrum München, Neuherberg, Germany. ${ }^{3}$ German Center for Diabetes Research (DZD), Neuherberg, Germany. ${ }^{4}$ Biological Research Pharmacology Department, Sun Pharma Advanced Research Company Ltd., Vadodara, India. ${ }^{5}$ Division of Metabolic Diseases and 6 Neurobiology of Diabetes, School of Medicine, Technical University of Munich, Munich, Germany. ${ }^{7}$ Molecular EXposomics and ${ }^{8}$ Institute of Experimental Genetics and German Mouse Clinic, Helmholtz Zentrum München, Neuherberg, Germany. ${ }^{9}$ Institute for Diabetes Research and Metabolic Diseases of the Helmholtz Zentrum München at the University of Tübingen, Tübingen, Germany. ${ }^{10}$ Department of Endocrinology and Metabolism, Amsterdam University Medical Center, University of Amsterdam, Amsterdam, Netherlands. "Netherlands Institute for Neuroscience, an Institute of the Royal Netherlands Academy of Arts and Sciences, Amsterdam, Netherlands. ${ }^{12}$ Institute of Developmental Genetics, Helmholtz Zentrum München, Neuherberg, Germany. ${ }^{3}$ Department of Developmental Genetics, School of Life Sciences Weihenstephan, Technical University of Munich, Munich, Germany. ${ }^{14}$ Lund University Diabetes Centre, Clinical Research Centre, Skåne University Hospital Malmö, Malmö, Sweden. ${ }^{15}$ YYNLAB Analytics and Services, Switzerland AG, Dielsdorf, Switzerland. ${ }^{16}$ Université de Paris, BFA, UMR 8251, CNRS, Paris, France. " ${ }^{17}$ Division of Endocrinology, Diabetology and Nephrology, Department of Internal Medicine IV, University of Tübingen, Tübingen, Germany. ${ }^{18}{ }^{C}$ Chair of Experimental Cenetics, School of Life Sciences Weihenstephan, Technical University of Munich, Freising, Germany. ${ }^{19}$ nstitute of Experimental and Clinical Pharmacology and Toxicology, Department of Pharmacology and Experimental Therapy, Eberhard Karls University Hospitals and Clinics, Tübingen, Germany. ${ }^{20}$ Cerman Center for Neurodegenerative Diseases (DZNE), Munich, Germany. ${ }^{21}$ Munich Cluster for Systems Neurology (SyNergy), Munich, Germany. ${ }^{22}$ Czech Centre for Phenogenomics, Institute of Molecular Genetics of the Czech Academy of Sciences, Vestec, Czech Republic. ${ }^{23}$ Department of Physiology, Instituto de Investigación Sanitaria, University of Santiago de Compostela, Santiago de Compostela, Spain. ${ }^{24}$ Department of Pediatrics, University of Cincinnati, Cincinnati, Ohio, USA. ${ }^{25}$ Howard Hughes Medical Institute, Cincinnati Children's Hospital Medical Center, Cincinnati, Ohio, USA. ${ }^{2}{ }^{2}$ Neurobiology of Stress Resilience, Max Planck Institute of Psychiatry, Munich, Germany. ${ }^{27}$ Institute for Clinical Chemistry and Pathobiochemistry, Department for Diagnostic Laboratory Medicine, University Hospital Tübingen, Tübingen, Germany.

\begin{abstract}
Recent genome-wide association studies (CWAS) identified DUSP8, encoding a dual-specificity phosphatase targeting mitogen-activated protein kinases, as a type 2 diabetes (T2D) risk gene. Here, we reveal that Dusp8 is a gatekeeper in the hypothalamic control of glucose homeostasis in mice and humans. Male, but not female, Dusp8 loss-of-function mice, either with global or corticotropin-releasing hormone neuron-specific deletion, had impaired systemic glucose tolerance and insulin sensitivity when exposed to high-fat diet (HFD). Mechanistically, we found impaired hypothalamic-pituitary-adrenal axis feedback, blunted sympathetic responsiveness, and chronically elevated corticosterone levels driven by hypothalamic hyperactivation of Jnk signaling. Accordingly, global Jnk1 ablation, AAV-mediated Dusp8 overexpression in the mediobasal hypothalamus, or metyrapone-induced chemical adrenalectomy rescued the impaired glucose homeostasis of obese male Dusp8-KO mice, respectively. The sex-specific role of murine Dusp8 in governing hypothalamic Jnk signaling, insulin sensitivity, and systemic glucose tolerance was consistent with functional MRI data in human volunteers that revealed an association of the DUSP8 rs 2334499 risk variant with hypothalamic insulin resistance in men. Further, expression of DUSP8 was increased in the infundibular nucleus of T2D humans. In summary, our findings suggest the GWAS-identified gene Dusp8 as a novel hypothalamic factor that plays a functional role in the etiology of T2D.
\end{abstract}

Conflict of interest: MHT is a member of the scientific advisory board of ERX Pharmaceuticals, Inc. He was a member of the Research Cluster Advisory Panel (ReCAP) of the Novo Nordisk Foundation between 2017 and 2019. He attended a scientific advisory board meeting of the Novo Nordisk Foundation Center for Basic Metabolic Research in 2016. He received funding for his research projects from Novo Nordisk (2016-2020) and Sanofi-Aventis (2012-2019). He was a consultant for Bionorica SE (2013-2017), Menarini Ricerche S.p.A. (2016), and Bayer Pharma AG Berlin (2016). As former Director of the Helmholtz Diabetes Center and the Institute for Diabetes and Obesity at Helmholtz Zentrum München (2011-2018) and since 2018 as CEO of Helmholtz Zentrum München he has been responsible for collaborations with a multitude of companies and institutions, including, but not limited to, Boehringer Ingelheim, Eli Lilly, Novo Nordisk, Medigene, Arbormed, and BioSyngen. In this role, he was/is further responsible for commercial technology transfer activities of his institute(s), including diabetes-related patent portfolios of Helmholtz Zentrum München, e.g., WO/2016/188932 A2 or WO/2017/194499 A1. Copyright: (๖) 2020, American Society for Clinical Investigation.

Submitted: January 14, 2020; Accepted: July 31, 2020; Published: October 12, 2020

Reference information: / Clin Invest. 2020;130(11):6093-6108.

https://doi.org/10.1172/JCl136363. 


\section{Introduction}

Obesity-induced insulin resistance is associated with an increase in circulating levels of cytokines and free fatty acids (1) that activate proinflammatory signaling pathways. Mitogen-activated protein kinase (MAPK) signaling plays a major role in this inflammatory process via sequential, dual phosphorylation and thereby activation of the serine/threonine kinases c-Jun $\mathrm{N}$-terminal kinase (Jnk), p38, and extracellular signal-regulated kinase (ERK) (2). The magnitude and duration of MAPK signaling dictates the specificity of signal transduction and the extent of biological signaling response (3). MAPKs are thus tightly controlled by MAPK phosphatases (MKPs) that form the structurally distinct family of dual-specificity phosphatases (DUSPs) (4). The DUSPs show high homology in an amino-terminal noncatalytic domain and in a carboxyl-terminal catalytic domain, but differ in their substrate specificities, tissue distribution, subcellular localization, and inducibility by extracellular stimuli (reviewed in refs. 3,5 ). Recent work using genetic models has revealed potentially important roles of DUSPs in the regulation of pathophysiological signaling with relevance to human diseases including cancer, diabetes, inflammatory, and neurodegenerative disorders (4). Here, the cytoplasmic, ERK-specific Dusp6 and Dusp9 and the inducible nuclear and promiscuous, i.e., ERK-, JNK-, and p38-dephosphorylating Dusp1 were reported to modulate glucose homeostasis. Less is known about MKPs specific for JNK and/or p38, namely Dusp8, Dusp10, and Dusp16 given their expression in both the cytoplasm and cell nucleus and their relatively selective substrate specificity $(4,6,7)$. Dusp8 appears to be of particular importance for the control of glucose homeostasis based on recent genomewide association studies (GWAS) that linked minor allele carriers of the SNP rs2334499 on chromosome 11p15, a region containing DUSP8 among a number of other genes, with a moderately increased risk for type 2 diabetes (T2D) $(8,9)$.

Dusp 8 was originally identified as an immediate early gene, and its expression can be stimulated in vitro by nerve growth factor and insulin (10). Expression of DUSP8 was found to be strong in adult human brain and weak in heart and skeletal muscle (10). Dusp8 overexpression studies revealed greater phosphatase activity against Jnk than p38 or ERK (11). Here, we aimed to delineate the functional importance of Dusp8 for the etiology of T2D. We show that DUSP8 polymorphism rs2334499 is associated with hypothalamic insulin resistance in men and that DUSP8 expression is increased in the infundibular nucleus of humans with T2D. Phenotypic studies in global Dusp8-KO mice and in Dusp8-KO mice with the hypothalamic rescue of Dusp 8 expression expose a specific role of Dusp8 as gatekeeper for systemic glucose tolerance and insulin sensitivity in an obesogenic environment. Mechanistic studies in Jnk1-Dusp 8 double-KO mice and mice with conditional deletion of Dusp 8 in corticotropinreleasing hormone $(\mathrm{CRH})$ neurons further revealed that Dusp8 as Jnk-specific phosphatase acts as a regulator for hypothalamic Jnk activation and hypothalamic-pituitary-adrenal (HPA) axis feedback inhibition that is essential for systemic glucose homeostasis.

\section{Results}

Expression levels of Dusp8 are upregulated in the hypothalamus of mice by body adiposity. We first aimed to investigate the murine expression pattern of $D u s p 8$, the product of a gene recently linked with an increased risk for T2D in humans $(8,9)$. Focusing on tissues relevant for glucose tolerance in mice, we largely confirmed earlier reports showing high expression levels of DUSP8 in adult human brain, heart, and skeletal muscle (10). Specifically, we showed high expression levels of Dusp 8 in several brain areas including the hypothalamus, and considerable expression in selected types of skeletal muscle of mice (Figure 1A). We next assessed the impact of nutrient overload on Dusp8 gene expression in vivo in HFDinduced obese (DIO) mice. Dusp 8 expression was not altered in muscle (Figure 1B) but significantly increased in hypothalami of DIO mice (Figure 1C). In contrast, prolonged fasting, which is known to mobilize massive amounts of free fatty acids into circulation, and refeeding by fat-free diet (FFD) or HFD had no impact on hypothalamic Dusp8 gene expression (Figure 1D). Moreover, hypothalamic Dusp 8 expression was also increased in genetically obese, chow-fed $L e p^{o b}$ mice (Figure $1 \mathrm{E}$ ) but unaffected by leptin replacement therapy (data not shown). These data indicate that the transcriptional regulation of $D u s p 8$ in mice is independent from leptin signaling or dietary fatty acids. In situ hybridization revealed that Dusp8 expression is increased in the mediobasal hypothalamus $(\mathrm{MBH})$, a region known for the regulation of glucose and energy homeostasis, of $L e p^{o b}$ mice compared with WT controls (Figure 1, F-H). Overall, we show that hypothalamic Dusp 8 expression appears to be regulated by body adiposity, a metabolic state that often correlates with impaired glucose homeostasis.

Global ablation of Dusp8 exacerbates HFD-induced glucose intolerance specifically in male mice. To explore whether Dusp8 has indeed a gluco- and energy-regulatory function in vivo we studied mice with global ablation of Dusp8. Male and female Dusp8KO mice that were fed a standard chow diet neither showed a difference in body weight (BW) or body composition nor displayed glucose or insulin intolerance compared with their respective WT controls (Supplemental Figure 1; supplemental material available online with this article; https://doi.org/10.1172/JCI136363DS1). After inducing nutrient overload and obesity by 12 weeks of HFD feeding, we found comparable changes in BW and body composition (Figure 2, A and B) as well as unperturbed food intake, energy expenditure, respiratory exchange ratios, locomotor activity, and leptin sensitivity in male Dusp8-WT and -KO mice (Supplemental Figure 2). However, despite similar propensities for DIO, male Dusp8-KO mice exposed to HFD showed an impairment of glucose homeostasis in a glucose tolerance test (GTT) (Figure 2, C and D). In a pyruvate tolerance test (PTT), we found increased baseline glucose levels after overnight fasting and impaired glucose clearance in HFD-fed Dusp8-KO males (Figure 2, E and F). An insulin tolerance test (ITT) revealed a decrease in insulin sensitivity in obese Dusp8-KO males (Figure 2, G and $\mathrm{H}$ ). Male mice heterozygous for the Dusp8 deletion showed similar glucose tolerance and insulin sensitivity as WT littermates after HFD exposure (data not shown). In male HFD-fed Dusp8-KO mice fasting plasma insulin levels (WT, $36.5 \pm 12.0 \mu \mathrm{U} / \mathrm{mL} ;$ Dusp $8-\mathrm{KO}, 67.0 \pm 10.4 \mu \mathrm{U} / \mathrm{mL}$ ) and homeostatic model assessment of insulin resistance (HOMA-IR) (Figure 2I) indices were significantly higher compared with their WT counterparts. Plasma markers for lipid metabolism (Supplemental Figure 3, A-C) and hepatic triglyceride stores (Supplemental Figure 3D) were comparable between HFD-fed 
A

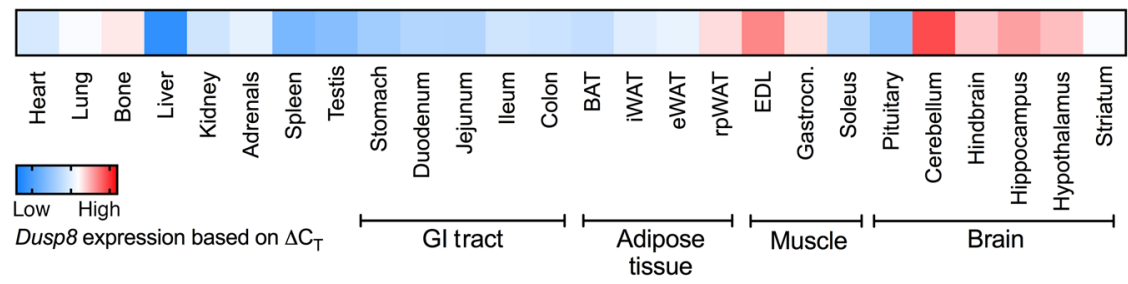

B

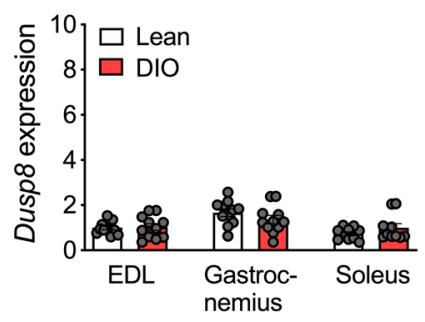

C
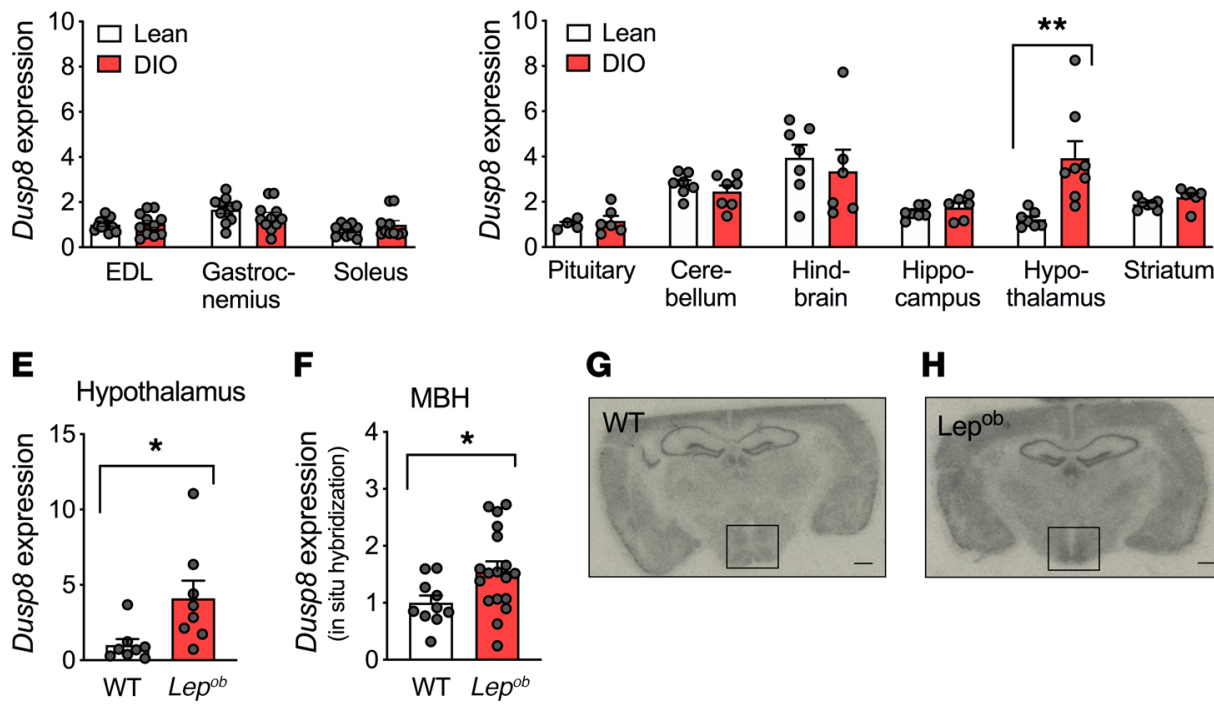

$F$

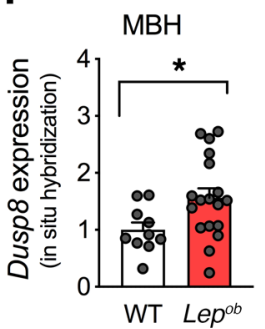

G

H

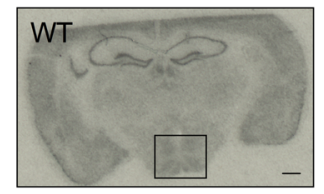

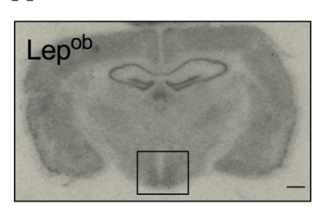

D

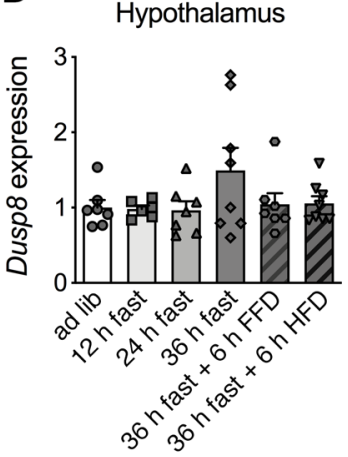

Figure 1. Hypothalamic Dusp8 expression is regulated by body adiposity. (A) Tissue-specific expression levels of Dusp8 were assessed in lean [567BL/6) male mice $(n=3)$ and are presented as $\Delta \mathrm{C}_{\mathrm{T}}$ values with low Dusp8 expression in blue and high Dusp8 expression in red. Regulation of Dusp8 expression by adiposity was analyzed in lean vs. diet-induced obese (DIO) male mice for (B) skeletal muscle (extensor digitorum longus, EDL $[n=12 \mathrm{WT}, n=11 \mathrm{KO}$ ], gastrocnemius [ $n=11 \mathrm{WT}, n=$ $12 \mathrm{KO}$ ], soleus [ $n=10 \mathrm{WT}, n=12 \mathrm{KO}]$ ) and (C) microdissected brain regions (pituitary [ $n=4 \mathrm{WT}, n=6 \mathrm{KO}$ ], cerebellum $[n=6 \mathrm{WT}, n=6 \mathrm{KO}$ ], hindbrain [ $n=6 \mathrm{WT}, n=5$ $\mathrm{KO}$, hippocampus [ $n=5 \mathrm{WT}, n=6 \mathrm{KO}$ ], hypothalamus [ $n=6 \mathrm{WT}, n=6 \mathrm{KO}]$, and striatum [ $n=6 \mathrm{WT}, n=5 \mathrm{KO}]$ ) and is shown as $\Delta \mathrm{C}_{\mathrm{T}}$ values. iWAT, inguinal white adipose tissue; eWAT, epididymal white adipose tissue; rpWAT, retroperitoneal white adipose tissue. (D) Hypothalamic Dusp8 mRNA expression in C57BL/6) mice that were subjected to $12(n=6), 24(n=7)$, or $36(n=8)$ hours of fasting as well as refeeding for 6 hours with fat-free (FFD, $n=7)$ or high-fat (HFD, $n=8)$ diet compared with ad libitum-fed control $(n=7)$. (E) Hypothalamic Dusp8 mRNA levels in male Lepobvs. WT mice $(n=8)$. (F) Densitometric analysis of Dusp8 mRNA by in situ hybridization localized Dusp8 expression to the MBH of male (G) WT $(n=10)$ and $(\mathbf{H})$ Lep ${ }^{\circ b}$ mice $(n=18)$ mice; representative pictures are shown. Data are shown as heatmap (A) or as scatter dot plots with means \pm SEM (B-F). Scale bars: $500 \mu \mathrm{m}$. ${ }^{*} P<0.05 ;{ }^{* *} P<0.01$ by Student's $t$ test (B, C, E, and F) or 1-way ANOVA (D).

Dusp8-WT and -KO males. An intravenous (i.v.) GTT revealed higher insulin secretion in obese Dusp8-KO males compared with WT controls (Figure 2, J and K), which points toward insulin resistance in Dusp8-KO males. In ex vivo analyses in isolated $\beta$ cells of male Dusp8-WT and -KO mice, we found comparable insulin content (Supplemental Figure 3E) and glucose-stimulated insulin secretion (Supplemental Figure 3F). Combined, these data suggest impaired insulin sensitivity instead of insulin secretory problems in HFD-fed Dusp8-KO males. Female Dusp8WT and -KO mice showed comparable susceptibilities to gain BW (Supplemental Figure 3G) and body fat (Supplemental Figure $3 \mathrm{H}$ ) when fed HFD. Similarly, neither glucose tolerance nor insulin sensitivity were affected by Dusp8 ablation in HFD-fed female mice (Supplemental Figure 3, I-L). Taken together, these data indicate impaired glucose tolerance and insulin resistance in male but not female Dusp8-KO mice exposed to HFD.

Glucose intolerance in obese, global Dusp8-KO mice can be reversed by hypothalamic Dusp8 overexpression. Next, we aimed to assess the specific role of hypothalamic Dusp8 by injecting Dusp8- overexpressing adeno-associated virus (AAV) into the MBH of WT mice, resulting in a 4 -fold higher hypothalamic Dusp 8 expression, compared with controls injected with green fluorescent proteinAAV (GFP-AAV) (Figure 2, L and M). AAV-mediated overexpression of Dusp8 in the MBH had no effect on glucose tolerance in HFD-fed WT male mice compared with GFP controls (Figure 2, $\mathrm{N}$ and O). However, overexpression of Dusp8 in the MBH of global Dusp8-KO males significantly improved their impaired glucose tolerance and normalized their glucose levels to those of HFD-fed WT males (Figure 2, N and O). None of the groups displayed changes in BW or body composition in response to AAV treatment (data not shown). The rescue of glucose intolerance by AAVmediated Dusp8 overexpression in the MBH points toward a central hypothalamic role of Dusp8 in regulating glucose homeostasis in an obesogenic environment.

Impaired hypothalamic insulin sensitivity, elevated glucocorticoid signaling, and reduced sympathetic responsiveness in HFD-fed Dusp8-KO males. The impaired glucose tolerance in Dusp8-KO males was reflected by aberrant expression levels of key enzymes 
A

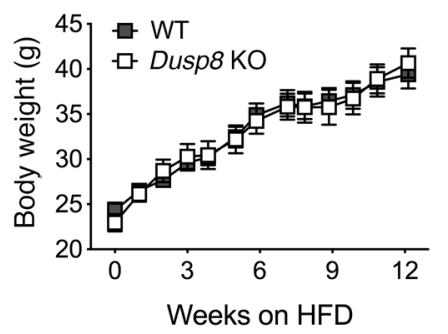

E

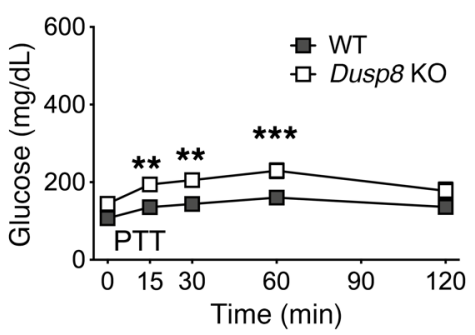

B

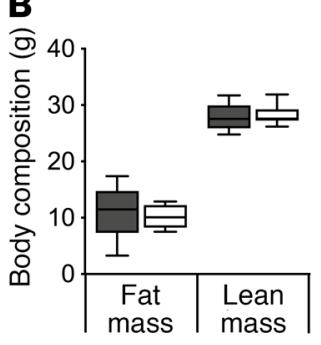

$\mathbf{F}$

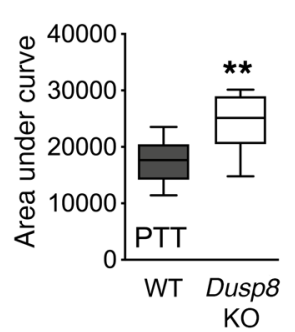

C

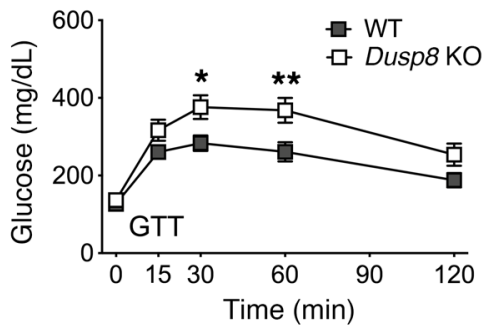

G

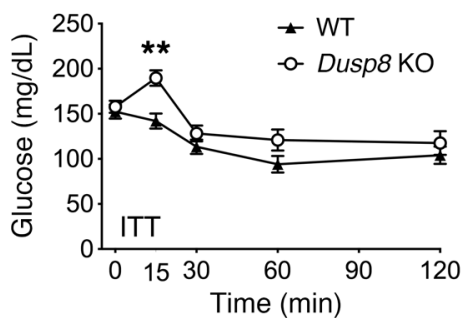

D

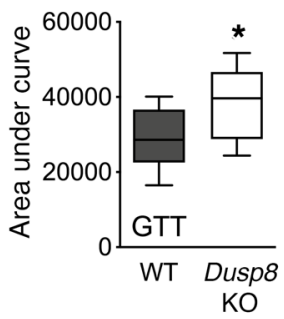

H

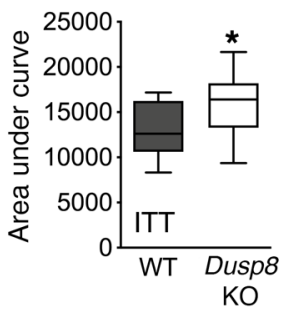

I

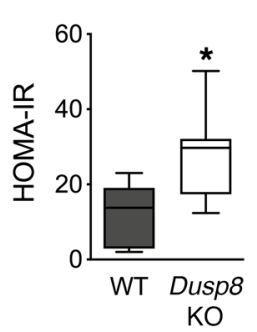

J
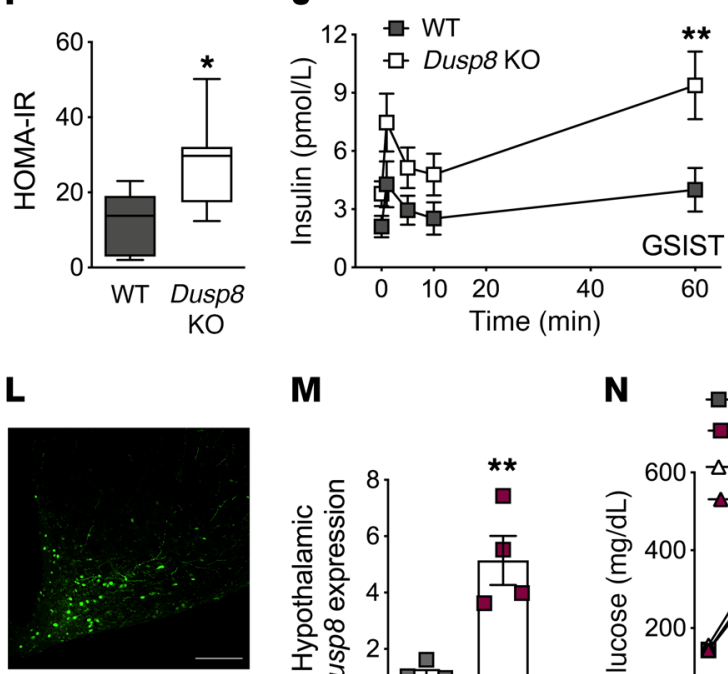

M

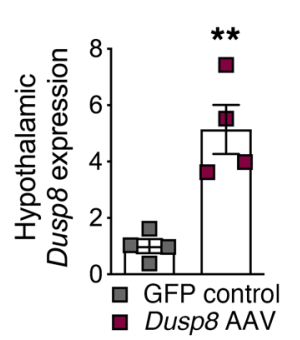

$\mathbf{N}$

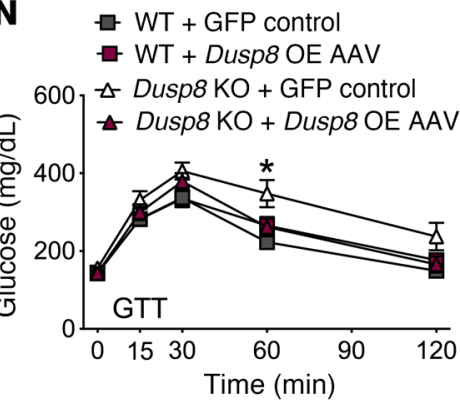

K

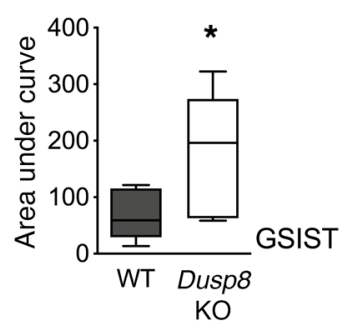

o

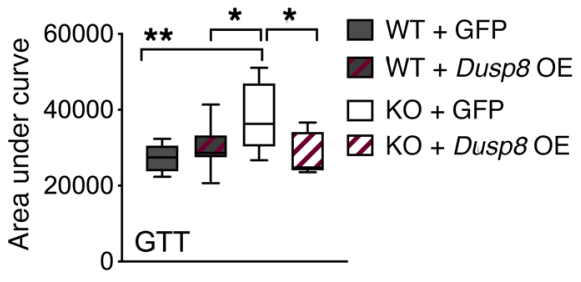

Figure 2. HFD-induced glucose intolerance in global male Dusp8-KO mice is reversed by hypothalamic Dusp8 overexpression. Male Dusp8-WT ( $n=14$ ) and $-\mathrm{KO}(n=9)$ littermates were subjected to $45 \%$ HFD feeding and evaluated for (A) body weight gain over 12 weeks and for (B) fat mass and lean mass after 13 weeks of HFD exposure. (C-H) Glucose tolerance (GTT), pyruvate tolerance (PTT), and insulin tolerance (ITT) tests were carried out after 12 weeks, 14 weeks, or 13 weeks of diet exposure, respectively ( $n=14$ WT, $n=9$ Dusp8-KO). (I) After 16 weeks of HFD exposure, fasting glucose and insulin levels were measured in male Dusp8-WT $(n=5)$ and -KO mice $(n=7)$ to calculate the HOMA-IR as (insulin [mg/dL] $\times$ glucose [mg/dL])/405. (J and K) Insulin secretion during an i.v. GTT was measured in an additional cohort of male Dusp8-WT and -KO mice ( $n=6$ each; 17 weeks HFD). (L) Representative picture of GFPAAV control virus injected into the MBH. (M) Hypothalamic Dusp8 mRNA expression 1 week after AAV-mediated overexpression of Dusp8 or control GFP in the MBH of lean WT mice $(n=4)$. ( $\mathbf{N}$ and $\mathbf{0})$ GTTs were carried out 4 weeks after AAV injections into the MBH of male Dusp $8-\mathrm{WT}$ and $-\mathrm{KO}$ mice $(n=10 \mathrm{WT}+$ GFP-AAV, $n=10$ WT + Dusp8-overexpressing [Dusp8-OE] AAV, $n=8$ Dusp8-KO + GFP-AAV, $n=10$ Dusp8-KO + Dusp8-OE AAV). Data are shown as means \pm SEM (A, C, E, G, J, and $\mathbf{N}$ ), as box-and-whisker plots (B, D, F, H, I, K, and $\mathbf{0}$ ), or as scatter dot plot (M). Scale bar: $200 \mu \mathrm{m}$. ${ }^{*} P<0.05 ;{ }^{* *} P<0.01$; ${ }^{* * *} P<0.001$ by 2-way ANOVA (A, C, E, G, J, and $\mathbf{N}$ ), Student's $t$ test (B, D, F, H, I, K, and $\mathbf{M}$ ), or 1-way ANOVA (0).

involved in glucose uptake, glycolysis, glycogen synthesis, and glycogen breakdown in liver and skeletal muscle of HFD-fed Dusp8-KO mice, compared with WT controls (Figure 3, A and B). In an acute insulin challenge test, we moreover observed reduced hypothalamic Akt phosphorylation in HFD-fed Dusp8-KO males relative to WT controls, which indicates impaired hypothalamic insulin responsiveness (Figure 3, C and D). In the hypothala- mus, we further observed increased expression levels of arginine vasopressin (Avp), oxytocin (Oxt), and Crh. Elevated Crh mRNA levels in the hypothalamus were reflected by increased adrenocorticotropin hormone (Acth) and CRH receptor 1 (Crhr1) expression in the pituitary as well as steroid synthesis enzymes in the adrenals (Figure 3E). Expression levels of glucocorticoid signaling components in liver, muscle, and hypothalamus were also 
A

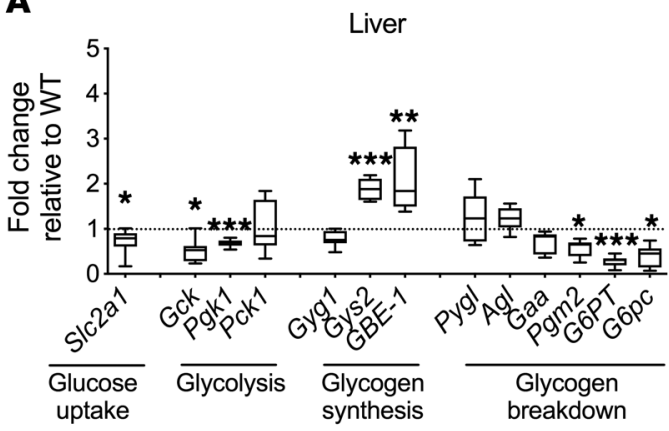

C

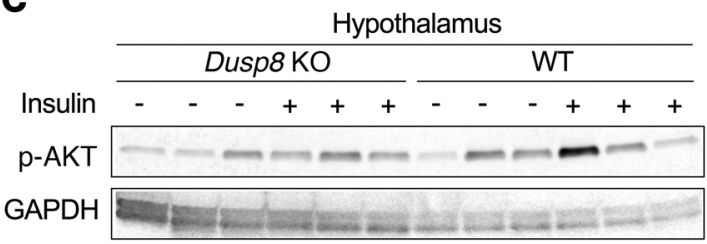

B

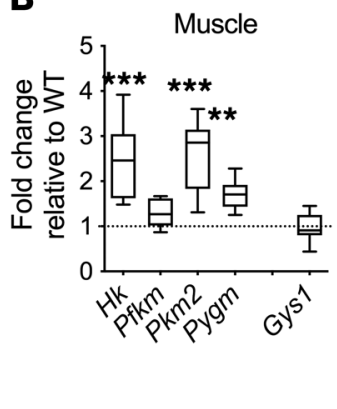

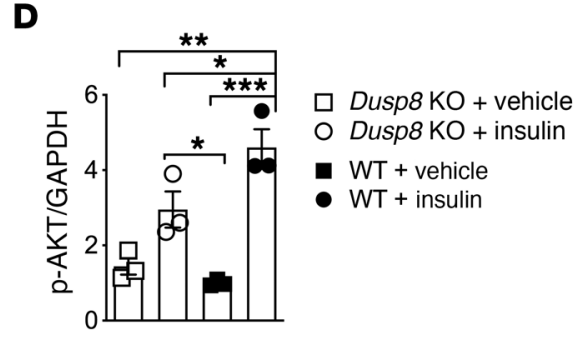

E

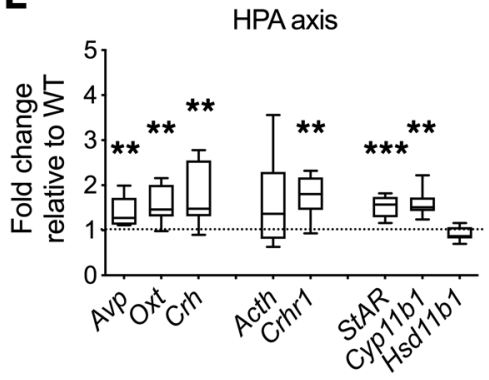

Hypothalamus $\overline{\text { Pituitary }} \overline{\text { Adrenals }}$
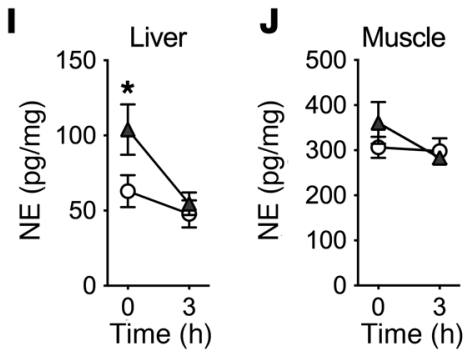

$\mathbf{F}$

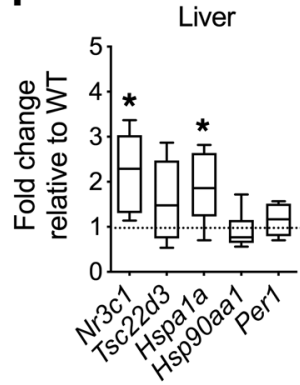

G

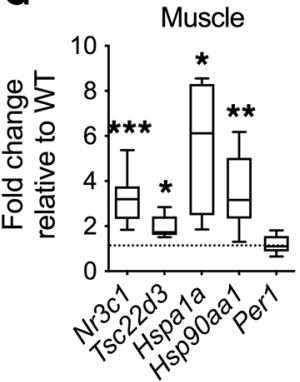

H
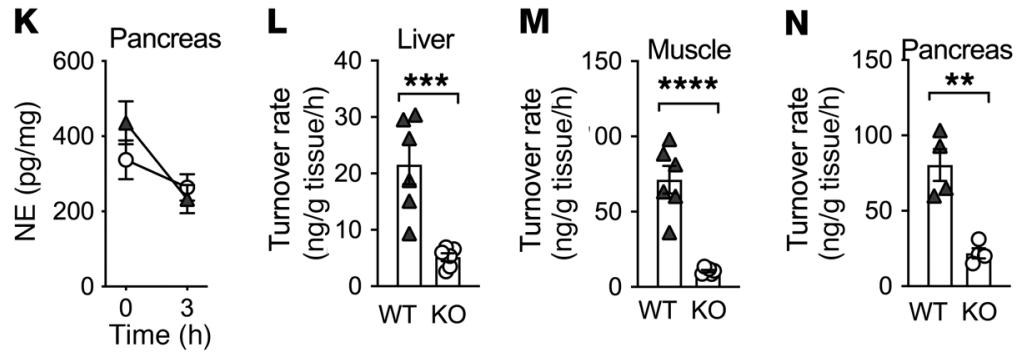

Figure 3. Elevated glucocorticoid action and impaired hypothalamic insulin sensitivity and sympathetic responsiveness in HFD-fed Dusp8-KO males. (A) Gene transcripts of hepatic enzymes involved in glucose metabolism ( $n=7 \mathrm{WT}, n=8$ Dusp8-KO) and (B) glucoregulatory enzymes in skeletal muscle ( $n=8$ WT, $n=8$ Dusp8-KO) in 17-week HFD-fed, male Dusp8-KO mice relative to WT controls. (C) Representative Western blot and (D) densitometric analysis of phosphorylated Akt relative to GAPDH in hypothalami of HFD-fed (17 weeks) Dusp8-KO and WT males that were acutely injected with 3 IU insulin/kg BW or saline as control and sacrificed 8 minutes later $(n=3)$. (E) Gene transcripts related to HPA axis regulation measured in the hypothalamus ( $n=8 \mathrm{WT}, n=8$ Dusp8-KO) and pituitary ( $n=8 \mathrm{WT}, n=8$ Dusp8-KO) as well as enzymes involved in steroid synthesis measured in the adrenals ( $n=8 \mathrm{WT}$, $n=7$ Dusp8-KO) of 17-week HFD-fed mice. Tissue-specific glucocorticoid action in (F) liver, (C) muscle, and (H) hypothalamus ( $n=8$ WT, $n=8$ Dusp8-KO) analyzed by qPCR. (I-K) Norepinephrine (NE) tissue content before and 3 hours after $\alpha$-MPT injection and (L-N) NE turnover rates in the liver, soleus, and pancreas of male Dusp8-WT and -KO mice exposed to HFD for 16 weeks ( $n=6$ for liver and soleus, $n=4$ for pancreas). Data are shown as box-and-whiskerplots (A, B, and E-H), as scatter dot plots ( $\mathbf{D}$ and $\mathbf{L}-\mathbf{N})$, or as means \pm SEM $(\mathbf{I}-\mathbf{K}) .{ }^{*} P<0.05$; ${ }^{* *} P<0.01 ;{ }^{* *} P<0.001 ;{ }^{* * *} P<0.0001$ by Student's $t$ test $(\mathbf{A}$ B, E-H, and L-N), 1-way ANOVA (D), or 2-way ANOVA (I-K).

increased (Figure 3, F-H), thus indicating elevated glucocorticoid action in HFD-fed Dusp8-KO males.

Increased glucocorticoid action in humans has recently been associated with an impaired sympathetic outflow (12) and lower norepinephrine (NE) levels (13), thereby impeding systemic glu- cose control to systemically drive glucose intolerance (14). Consistent with these human data, we observed lower baseline NE levels in livers of Dusp8-KO males compared with WT mice (Figure 3I; 0 hours). In contrast, NE baseline levels were unaltered in soleus muscle and pancreas of Dusp8-KO mice (Figure 3, J and K; O hours). 
However, the application of a single dose of the tyrosine hydroxylase inhibitor $\alpha$-MPT (15) revealed decreased NE turnover rates in liver, muscle, and pancreas of Dusp8-KO mice (Figure 3, L-N). In contrast to the HFD-fed Dusp8-KO males, female Dusp8-KO mice did not display deregulated glucose metabolism or elevated glucocorticoid action when exposed to HFD (Supplemental Figure 4), which is in agreement with their unaffected glucose tolerance and insulin sensitivity. Overall, the data point toward blunted sympathetic responsiveness as a consequence of increased glucocorticoid action in HFD-fed male Dusp8-KO mice.

Blunted HPA axis feedback control in Dusp8-deficient male mice. Consistent with the elevated expression of glucocorticoid receptor (GR) target genes, we found increased basal corticosterone levels measured 2 hours after the onset of the light phase (9 am) in HFD-fed Dusp8-KO males compared with WT controls (Figure $4 \mathrm{~A})$. Increased glycogen storage in livers of HFD-fed Dusp8-KO mice (Figure 4B) and our recent finding of decreased hippocampal mass in Dusp8-KO males (16) were further indicators for chronic hypercorticosteronemia driven by Dusp8 deficiency. The absence of a marked adrenal hypertrophy in HFD-fed Dusp 8 -KO mice compared with WT controls (Figure 4C) suggested a dysfunctional HPA axis feedback at the level of the hypothalamus or the pituitary as cause for the observed increased basal corticosterone levels. A combined dexamethasone suppression/CRH stimulation test (Dex/CRH) (17) revealed diminished suppression of corticosterone production after low-dose dexamethasone administration in HFD-fed Dusp8-KO mice (Figure 4D). Peripheral injection of $\mathrm{CRH}$ induced a robust increase in corticosterone concentrations, which was significantly more pronounced in Dusp8-KO mice compared with WT controls. Taken together, these data indicate compromised GR-mediated negative feedback at the level of the hypothalamus and at the pituitary, possibly due to a hypothalamic CRH overdrive.

Chemical adrenalectomy normalizes glucose tolerance in HFD-fed Dusp8-KO mice. To determine whether hypercorticosteronemia is causally driving the observed glucose intolerance in HFD-fed Dusp8-KO mice, we blocked endogenous corticosterone production by metyrapone treatment (18). Following this widely used method of chemical adrenalectomy, we found a normalization of the glucose intolerance phenotype, with comparable glucose excursions and AUC levels for male WT and Dusp8-KO mice after 14 days of metyrapone treatment (Figure 4, E and F). Metyrapone treatment was further sufficient to normalize gene expression of key genes involved in T2D pathophysiology and multiple glucocorticoid target genes (Supplemental Figure 5, A-G). Prior to metyrapone treatment, we confirmed glucose intolerance in our cohort of HFD-fed Dusp8-KO mice compared with WT controls (Supplemental Figure 5, H and I). We furthermore found no effect of metyrapone treatment on BW (Supplemental Figure 5J). The normalization of glucose tolerance and peripheral glucoregulatory gene profiles by metyrapone treatment suggests that the elevated basal cortisol concentrations are causal for the dysregulation of glucose homeostasis in Dusp8-KO mice.

Hypercorticosteronemia and glucose intolerance in CRH neuronspecific Dusp8-KO mice. Given the increased hypothalamic expression of CRH and the blunted HPA axis feedback control indicating CRH overdrive from the hypothalamus in HFD-fed global Dusp8-
KO mice, we next investigated whether the conditional ablation of Dusp8 in CRH-producing neurons (Figure 4G) can recapitulate the glucose intolerance and hypercorticosteronemia phenotype of the global Dusp8-KO mice. Male Dusp8 $8^{\text {CRH-Cre }} \mathrm{WT}$ and KO mice had similar BW and body composition after exposure to HFD for 12 weeks (Figure 4, H and I). As hypothesized, ablation of Dusp8 specifically in CRH neurons indeed impaired glucose tolerance (Figure 4, $\mathrm{J}$ and $\mathrm{K}$ ) and insulin sensitivity (Figure 4, $\mathrm{L}$ and $\mathrm{M}$ ) in HFD-fed mice. Plasma insulin levels (WT, $267.7 \pm 58.5 \mu \mathrm{U} / \mathrm{mL}$; Dusp8-KO, $325.6 \pm 56.0 \mu \mathrm{U} / \mathrm{mL} ; P=0.0277$ ) and HOMA-IR (Figure $4 \mathrm{~N}$ ) indices were significantly higher in male Dusp $8^{\text {CRH-Cre }} \mathrm{KO}$ mice on HFD compared with their WT counterparts. Further, we found increased basal corticosterone levels in Dusp $8^{\text {CRH-Cre }} \mathrm{KO}$ mice compared with WT controls (Figure 4O).

Given that the impairments in glucose tolerance and insulin sensitivity appeared milder in the Dusp $8^{\text {CRH-Cre }} \mathrm{KO}$ mice compared with global Dusp 8 -KO mice, we hypothesized a glucoregulatory role of Dusp8 also in other neuronal subpopulations in the hypothalamus. We thus generated mice with a conditional ablation of Dusp8 in Agouti-related peptide (AgRP) or proopiomelanocortin (POMC) neurons. Both hypothalamic neuronal populations are essential for metabolic homeostasis (19), and interconnections of AgRP neurons with $\mathrm{CRH}$ neurons are moreover linked with stress regulation and energy homeostasis (20). However, we did not reveal any alterations in the susceptibilities to diet-induced weight or fat mass gain, glucose or insulin tolerance, or corticosterone levels between HFD-fed male Dusp $8^{\text {AgRP-Cre }}$ WT versus KO males (Supplemental Figure 6, A-G) or Dusp $8^{\text {POMC-Cre }}$ WT versus KO males (Supplemental Figure 6, $\mathrm{H}-\mathrm{N}$ ). Overall, these results suggest a prominent role of Dusp8 in $\mathrm{CRH}$ neurons in the regulation of HPA axis reactivity, plasma corticosterone levels, and glucose homeostasis.

Dusp8 is a gatekeeper for Jnk activity and Jnk-dependent regulation of GR activity. To identify the signaling pathway mediating the impaired glucose tolerance and HPA axis feedback inhibition in our Dusp8 loss-of-function mice, we initially assessed the phosphatase activity of Dusp8 toward the MAPKs Jnk, p38, and ERK using an in vitro model of Dusp8 overexpression. Exposure of cells to the general MAPK activator and ER stressor anisomycin showed that overexpression of Dusp8 abolished the phosphorylation of Jnk and partially that of p38 (Figure 5, A-D). Notably and despite recent evidence suggesting a role for Dusp8 in ventricular remodeling via altering ERK1/2 activity (21), we found unaltered ERK phosphorylation in HEK293 cells stimulated with anisomycin (Figure 5, A and E). Overall, these data indicate that Dusp8 shows highest phosphatase activity toward Jnk, weaker activity toward p38, and no activity toward ERK in cells stimulated by anisomycin.

In the past, Jnk hyperactivation was shown to impair GR activity via phosphorylation at the inhibitory-site Ser226 (22). First, we corroborated that anisomycin treatment increases Jnk activity as well as GR phosphorylation at the inhibitory Ser226 residue (Figure 5F). We then found that overexpression of Dusp8 abolishes both Jnk activation as well as $\mathrm{GR}^{\mathrm{Ser} 226}$ phosphorylation (Figure 5, $F$ and $G)$. In a GR luciferase reporter assay, we further revealed functional relevance for the protective role of Dusp8 in Jnkdriven $\mathrm{GR}^{\text {Ser226 }}$ phosphorylation. Overexpression of Dusp8 had no effect on basal, dexamethasone-induced stimulation of GR 
A

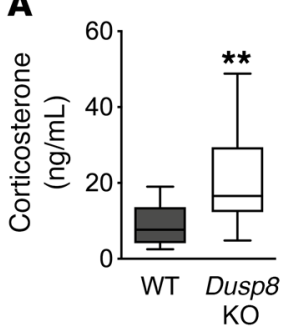

B

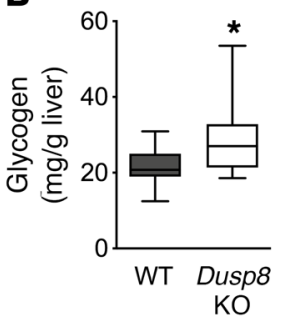

C

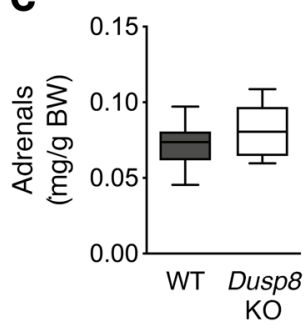

D

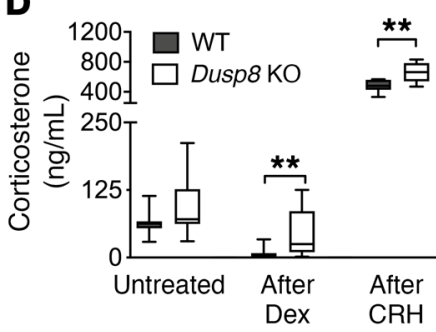

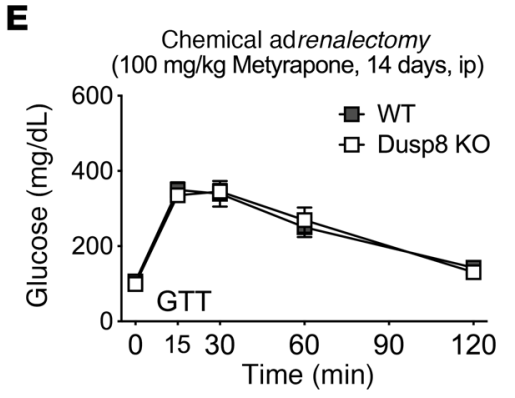

G

Dusp $8^{\text {tmala(EUCOMM) Hmgu }}$

$\mathbf{F}$

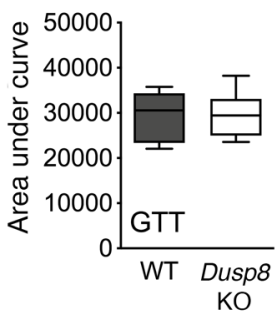

H

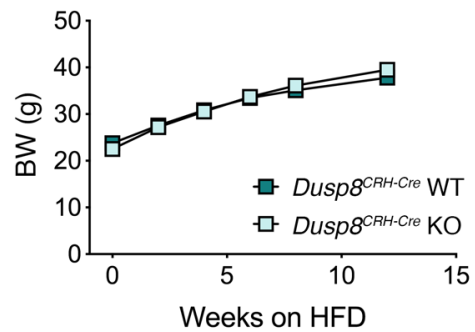

I

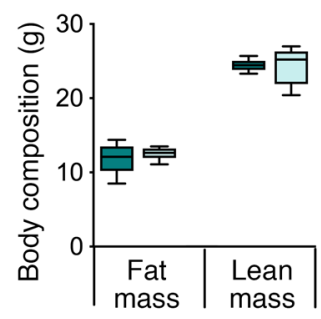

J

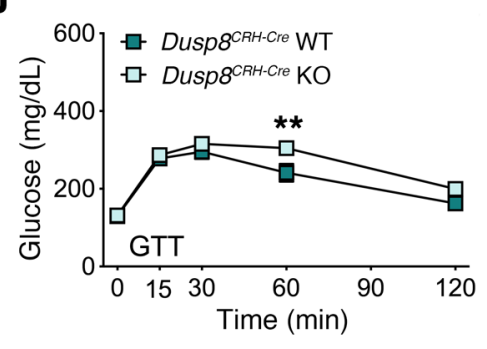

$\mathbf{K}$

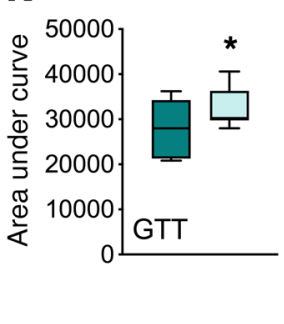

$\mathbf{L}$

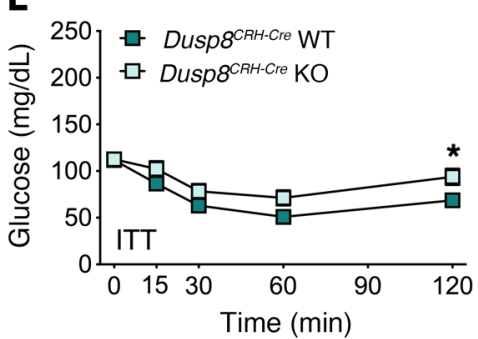

M

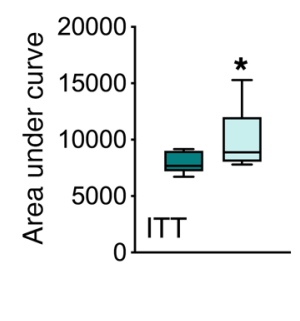

$\mathbf{N}$

o
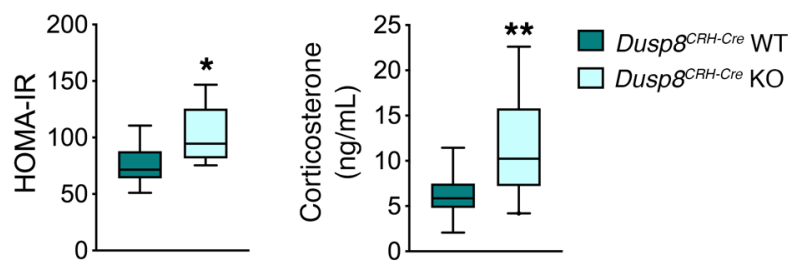

Figure 4. Chronic hypercorticosteronemia drives impaired glucose intolerance in HFD-fed male Dusp8-KO mice in a CRH neuron-specific manner. (A) Plasma corticosterone levels in male mice sacrificed under basal conditions at 9 am ( $n=11 \mathrm{WT}, n=12$ Dusp8-KO; 17 weeks HFD). (B) Liver glycogen levels and (C) adrenal weight normalized to body weight were determined after exposure to HFD for 17 weeks ( $n=15$ WT, $n=14$ Dusp8-KO). (D) Plasma corticosterone levels of HFD-fed Dusp8-KO $(n=11)$ and WT $(n=11)$ males that were subjected to a combined Dex/CRH test with pharmacological suppression of adrenocortical activity by dexamethasone $(0.05 \mathrm{mg} / \mathrm{kg} \mathrm{BW})$ and a subsequent stimulation with CRH (0.15 mg/kg BW). (E and F) Glucose tolerance test (GTT) was carried out after 14 days of daily metyrapone injections (i.p., $100 \mathrm{mg} / \mathrm{kg} \mathrm{BW}$ ) in male, HFD-fed Dusp8-WT $(n=5)$ and -KO $(n=6)$ mice. (G) Schematic for the generation of conditional, CRH neuron-specific Dusp8-KO mice. (H) Body weight gain over 12 weeks, and (I) fat mass and lean mass of

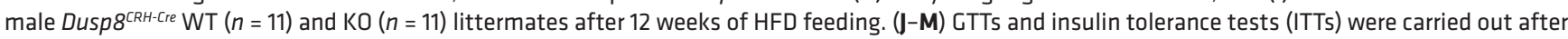

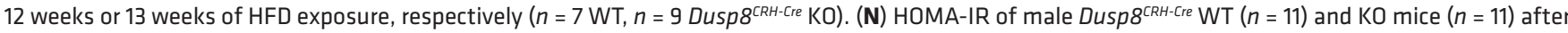
14 weeks of HFD exposure. (0) Plasma corticosterone levels of male Dusp8 ${ }^{\text {CRH-Cre } W T}(n=14)$ and KO mice $(n=20)$ after 14 weeks of HFD exposure. Data are shown as box-and-whisker plots (A-D, F, I, K, and $\mathbf{M}-\mathbf{0}$ ) or as means $\pm \mathrm{SEM}(\mathbf{E}, \mathbf{H}, \mathbf{J}$, and $\mathbf{L}) .{ }^{*} P<0.05 ;{ }^{*} P<0.01$ by Student's $t$ test $(\mathbf{A}-\mathbf{D}, \mathbf{F}, \mathbf{I}, \mathbf{K}$, and $\mathbf{M}-\mathbf{0})$ or 2-way ANOVA (E, H, J, and L). 
A

A + - + + $100 \mathrm{nM}$ Anisomycin

- + + - + + $250 \mathrm{nM}$ Anisomycin

$+++-{ }_{-}-$pCMV6

- + + + + pCMV6-Dusp8

- $=$ - $-\mathrm{p}$-Jnk

三ニニ三E三 $\mathrm{Jnk}$

삐 -

$-=-\ldots$ cJun

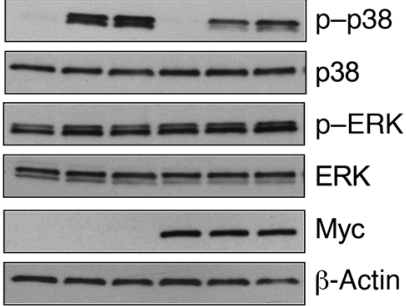

$-+\ldots 100 \mathrm{nM}$ Anisomycin

++++ pcDNA3.1-hGR

++- - pCMV6

- + + + pCMV6-Dusp8

$-\quad \longrightarrow$ p-S226 GR

- - Total GR

르.Jnk

Tenmen Jnk

$\ldots$ B-Actin

$J$

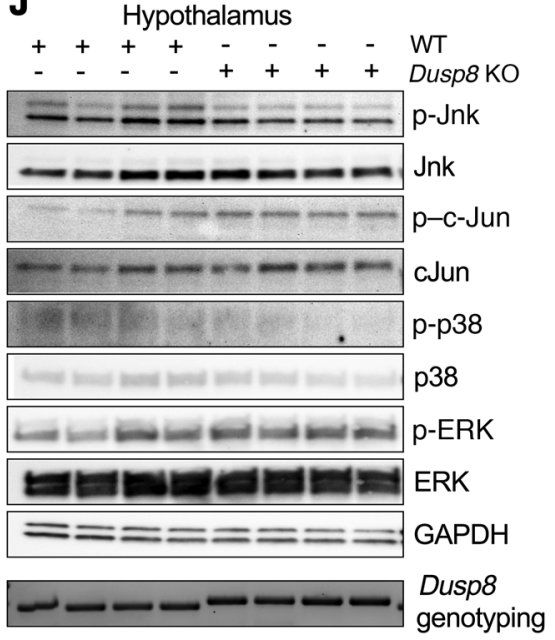

Q

Hypothalamus

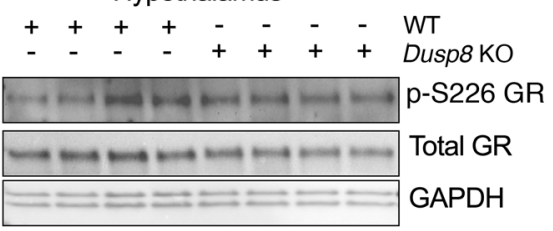

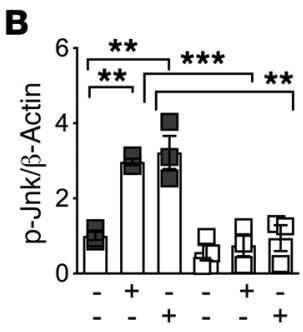
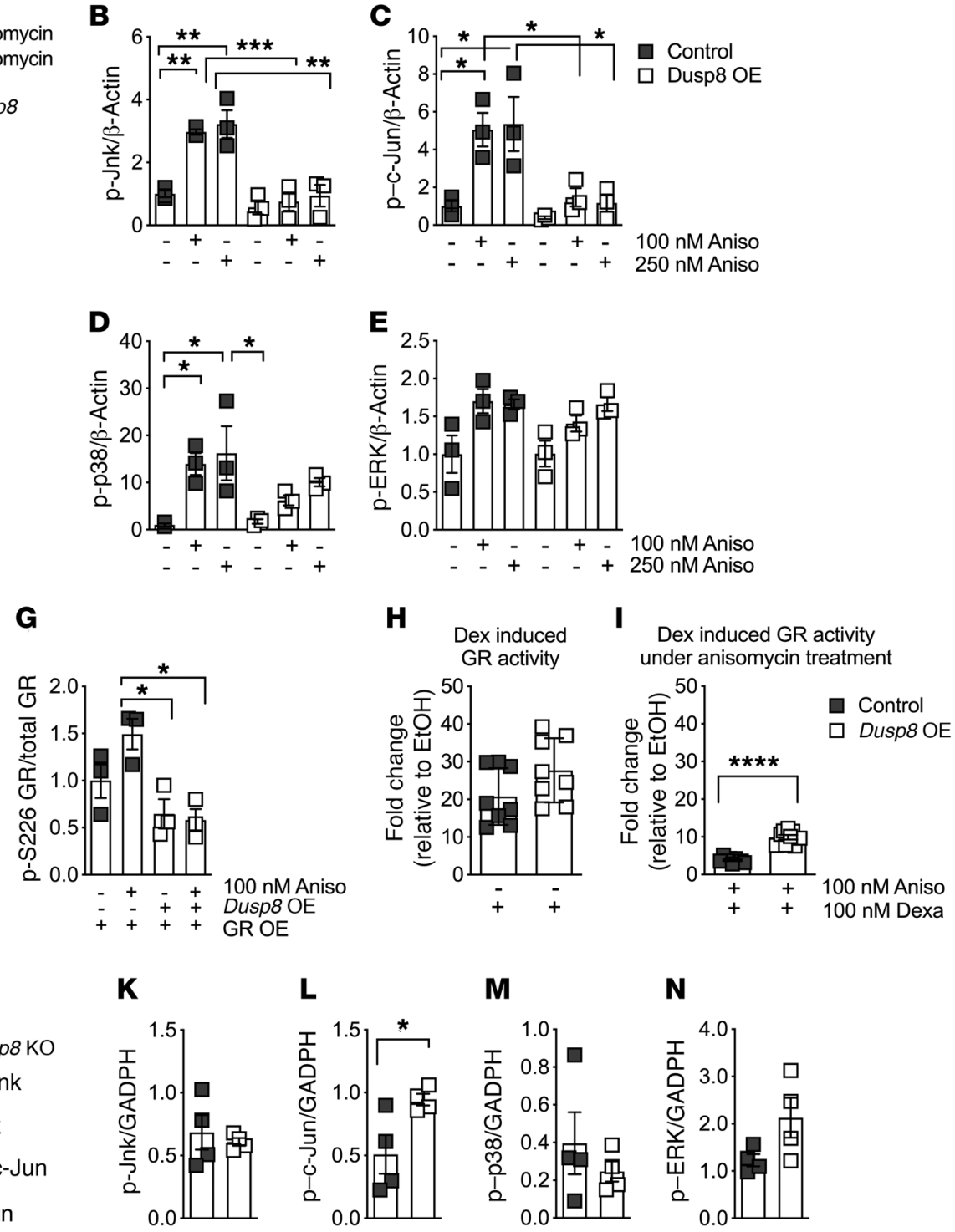

M
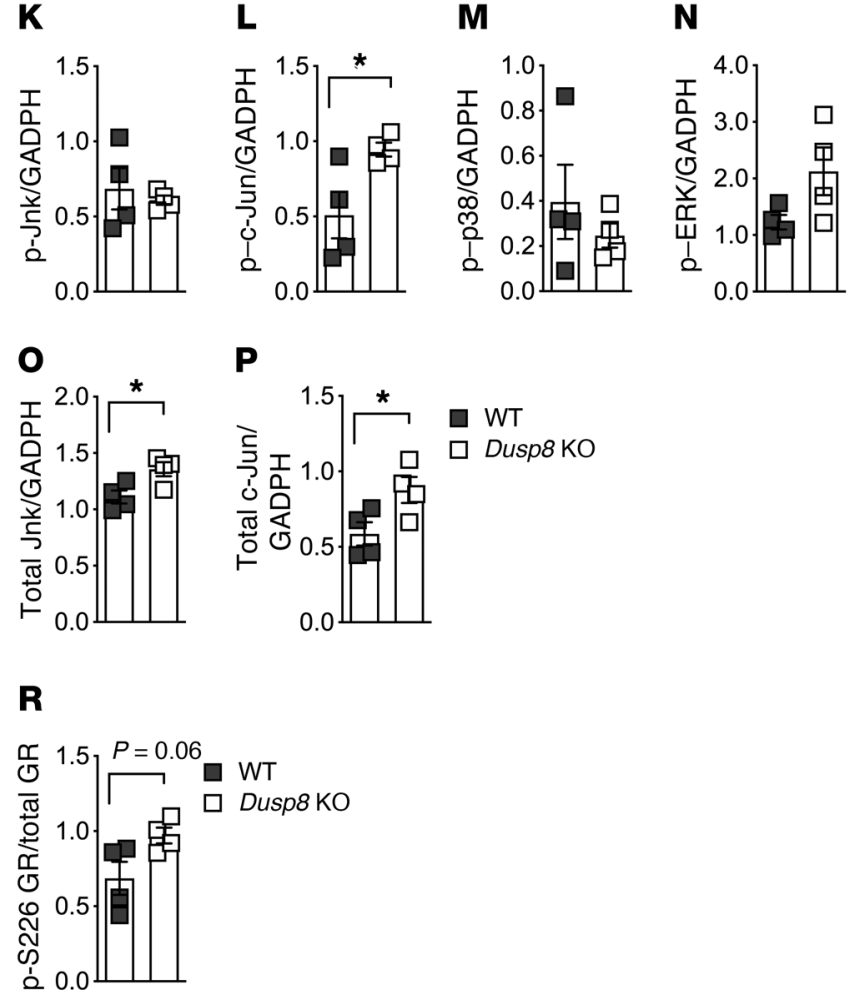
Figure 5. The Jnk-specific phosphatase Dusp8 ameliorates the inhibitory effect of Jnk signaling on glucocorticoid action. (A) Representative Western blot of an acute stimulation with the MAPK activator anisomycin (30 minutes) in HEK293 cells with Dusp8 overexpression (OE, confirmed by presence of $\mathrm{Myc}$ ), compared with pCMV6 control vector. Densitometric analysis of anisomycin-induced phosphorylation of (B) Jnk, (C) c-Jun, (D) p38, and (E) ERK relative to $\beta$-actin $(n=3)$. (F) Representative Western blot and C) densitometric analysis of HEK293 cells with hGR OE and/or Dusp8 OE that were stimulated with and without anisomycin (30 minutes) and then analyzed for phosphorylated GR at Ser226 relative to total GR $(n=3)$. (H) GR luciferase reporter assay activity in HEK293 cells overexpressing Dusp8 or an empty control plasmid stimulated with dexamethasone (5 hours, $n=3$ in biological triplicates). EtOH, ethanol. (I) Dexamethasone-induced GR luciferase reporter assay activity in HEK293 cells with Dusp8 OE that were pretreated with anisomycin (overnight, $n=3$ in biological triplicates). (J) Western blot of hypothalami of HFD-fed (16 weeks) male Dusp8-KO and WT mice ( $n=4$ each). Cenotypes were confirmed by PCR followed by agarose gel electrophoresis (WT = $370 \mathrm{bp}, \mathrm{KO}=430 \mathrm{bp}$ ). (K-P) Densitometric analysis of phosphorylated Jnk, c-Jun, p38, and ERK as well as total protein levels of Jnk and c-Jun relative to GAPDH ( $n=4 \mathrm{WT}$, $n=4 \mathrm{KO} ; 16$ weeks HFD). (Q) Western blot and (R) densitometric analysis of phosphorylated GR at Ser226 relative to total GR in hypothalami of male Dusp8-KO $(n=4)$ and WT mice $(n=4)$. Data are shown as scatter dot plots. ${ }^{*} P<0.05$; ${ }^{* *} P<0.01$; ${ }^{* *} P<0.001$; ${ }^{* * *} P<0.0001$ by 1-way ANOVA (B-E and $\mathbf{~ G ) ~ o r ~ S t u d e n t ' s ~} t$ test (H, I, K-P, and $\mathbf{R}$ ).

activity (Figure 5H). Addition of the stressor anisomycin dramatically repressed the transcriptional activity of GR (Figure 5I), but the Jnk-mediated downregulation of GR activity was ameliorated by Dusp8 overexpression (Figure 5I). These data indicate that a reduction in Jnk activity by Dusp8 signaling can augment GRdependent transcription.

Next, we assessed the effect of Dusp8 ablation on MAPK phosphorylation in our Dusp8-KO mouse model (Figure 5, J-P). Due to the unavailability of a specific antibody against Dusp8 (Supplemental Figure 7), genotypes were confirmed by PCR analysis. Phosphorylation of the Jnk downstream target c-Jun was upregulated in the hypothalamus of HFD-fed Dusp8-KO males compared with WT controls (Figure 5, J and L) along with elevated total protein levels of Jnk and c-Jun (Figure 5, J, O, and P). In muscle of HFD-fed mice the phosphorylation of MAPK was barely detectable (Supplemental Figure 8, A and B). In livers of Dusp8KO males we found increased phosphorylation of Jnk and c-Jun (Supplemental Figure 8, C and D), whereas no differences were detected for epididymal white adipose tissue (eWAT) (Supplemental Figure 8, E and F). In line with the in vitro results in cells, we found a trend toward increased GR phosphorylation at the inhibitory Ser226 residue in the hypothalamus of Dusp8-KO mice (Figure 5, Q and R). GR ${ }^{\text {Ser226 }}$ phosphorylation was significantly increased in muscle, but decreased in liver and unaltered in eWAT of Dusp8-KO mice (Supplemental Figure 8, G-L). Hypothalamic phospho-proteome analyses revealed an overall increase in the number of phosphorylated peptides in Dusp8-KO males compared with WT controls (Supplemental Figure 8M), but due to a relatively low abundance we could not detect any significantly deregulated MAPK phosphorylation sites in the hypothalamus of Dusp8-KO males (Supplemental Figure 8N and Supplemental Table 1). Combined, these results confirm substrate preference of Dusp8 for Jnk, but also indicate a putative crosstalk between MAPK. Overall, our data suggest an important protective role of Dusp8 as gatekeeper for hypothalamic Jnk signaling, the Jnkdependent negative feedback inhibition of hypothalamic GR signaling, and glucocorticoid action.

Jnk1 codeletion normalizes glucose tolerance and hypercorticosteronemia in HFD-fed Dusp8-KO mice. Hypothalamic phosphatase activity of Dusp8 toward Jnk was especially high in the arcuate nucleus (ARC), a major hypothalamic center governing energy and glucose homeostasis, measured by increased phosphorylation of c-Jun in HFD-fed mice with global ablation of Dusp8 compared with WT controls (Figure 6, A-C). The number of nuclei with phosphorylated c-Jun was also increased in the ARC of HFD-fed male Dusp $8^{\text {CRH-Cre }} \mathrm{KO}$ mice compared with WT controls (Supplemental Figure 9). Microarray analyses of laser-capture-microdissected ARC from HFD-fed global Dusp8-WT and -KO mice confirmed alterations in MAPK and GR signaling in Dusp8-KO males relative to WT controls (Figure 6, D and E, and Supplemental Table 2). We further observed an enrichment of deregulated genes involved in neuroinflammation, insulin action, and AMPK, LXR/RXR, PI3K, PKA, and STAT3 signaling. Single-cell expression data suggested a mostly neuronal role of Dusp8 (23), which was in line with the absence of microgliosis or astrocytosis in global Dusp8-KO males on HFD (Supplemental Figure 10).

To corroborate that glucose intolerance of Dusp8-KO males is driven by altered Jnk activity, we generated global Jnk1-Dusp8 double-knockout (dKO) mice. When fed chow diet, WT, Dusp8KO, Jnk1-KO, and Jnk1-Dusp8-dKO males displayed comparable BWs and body composition, as well as unchanged glucose tolerance and insulin sensitivity in all genotypes (Supplemental Figure 11). Upon exposure to HFD, Jnk1-KO mice were protected from DIO (Figure 6, F-H), as published previously (24). BWs and body composition of Jnk1-Dusp8-dKO mice on HFD were unaltered compared with Jnk1-KO mice but showed a tendency to be reduced when compared with Dusp8-KO mice and WT controls (Figure 6, F and G). Consistent with the high hypothalamic Jnk activity in Dusp8-KO males on HFD, the hypothalamic expression of inflammatory markers Emr1, Ikkb,ILIb, and $N f k b$ was increased in Dusp8-KO males on HFD but reversed when Jnk1 was codeleted in Dusp8-KO mice (Figure 6I). In line with the known role of hypothalamic Jnk as regulator of systemic glucose homeostasis (25), codeletion of Jnk1 in Dusp8-KO mice on HFD improved glucose tolerance (Figure 6, J and $\mathrm{K}$ ) and insulin sensitivity (Figure 6, L and M) compared with Dusp8-KO mice. The improvements in glucose homeostasis in Jnk1-Dusp8-dKO mice were reflected by improved fasting insulin (Figure $6 \mathrm{~N}$ ) and HOMA-IR values (Figure 6O) relative to Dusp8-KO mice. We further revealed that the increased Jnk activity in the global Dusp8-KO mice was causally driving the observed hypercorticosteronemia, as Jnk1-Dusp 8dKO mice on HFD showed normalized plasma corticosterone levels (Figure 6P).

Finally, as p38 MAPK is another potential target of Dusp8 (11), we coinserted a dominant-negative p38 allele into global Dusp8KO mice, which led to hypomorphic p38 activity (26), but had no effect on impaired glucose tolerance in HFD-fed Dusp 8 -KO mice (Supplemental Figure 12). Overall, our results demonstrate that codeletion of Jnk1 in Dusp8-KO mice reverses the hypothalamic inflammation and impaired glucose tolerance and insulin sensitivity observed in HFD-fed Dusp8-KO mice. This finding is con- 
A

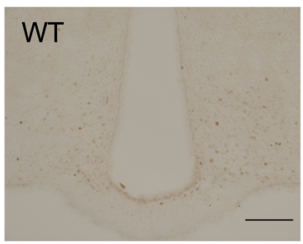

B

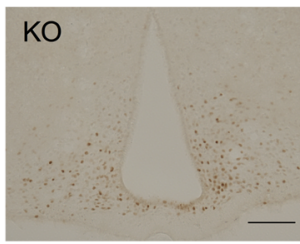

C

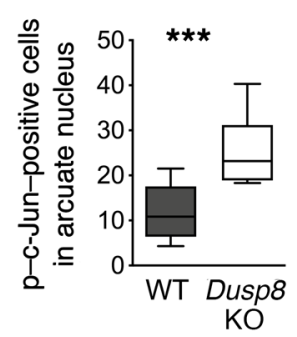

D

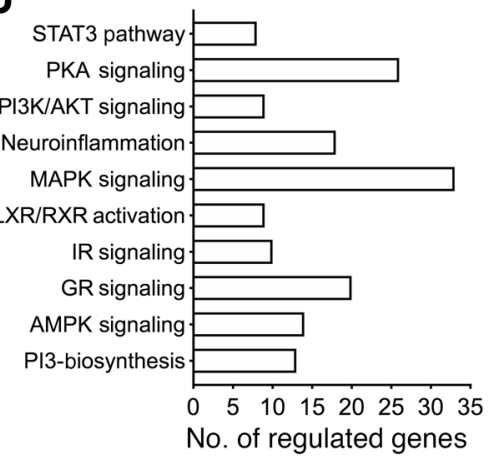

E

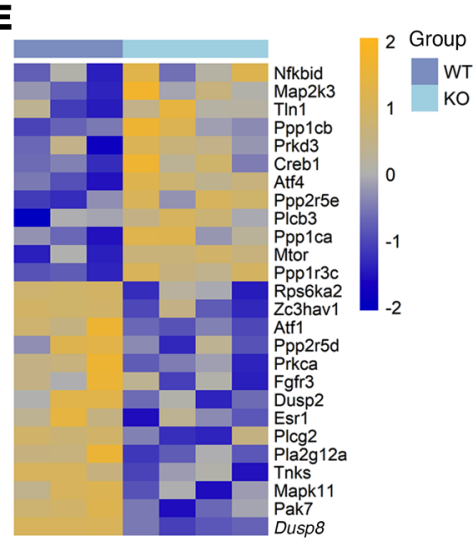

$\mathbf{F}$

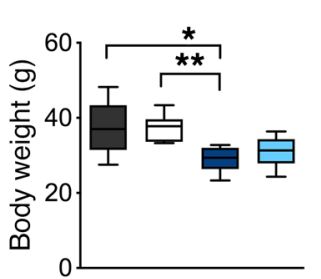

G

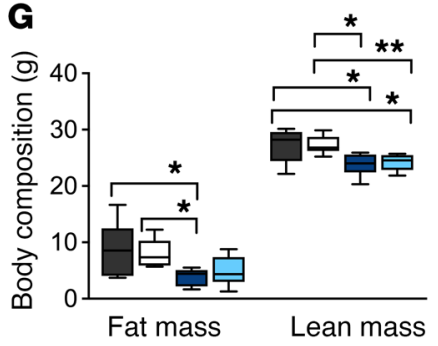

H

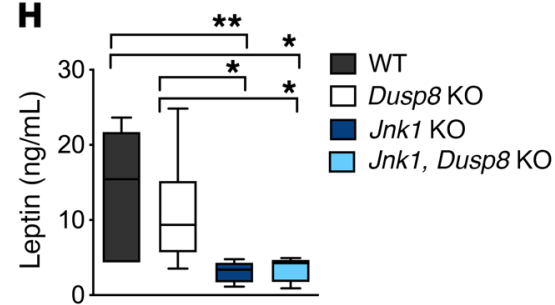

I

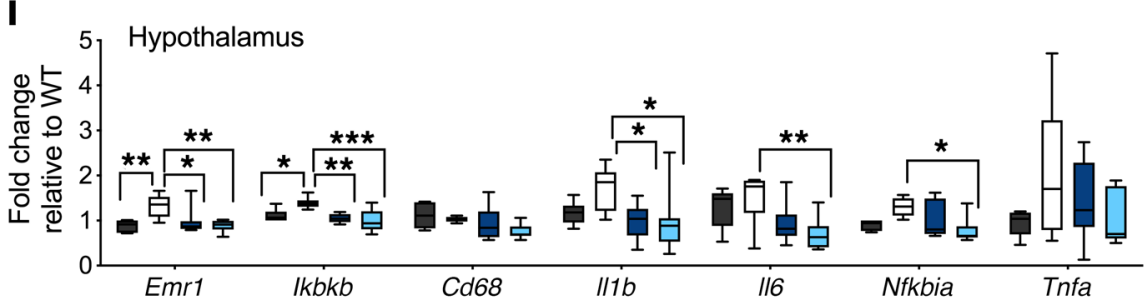

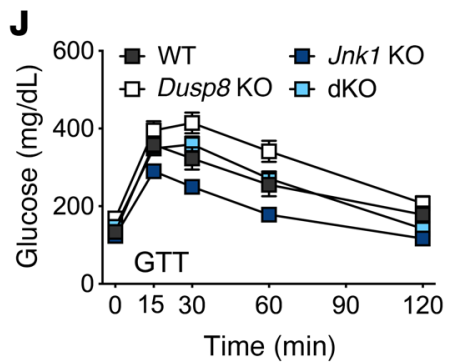

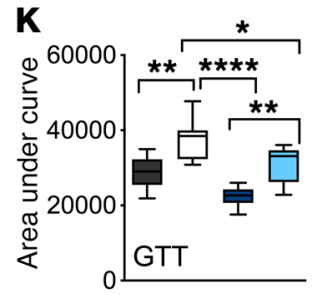

L

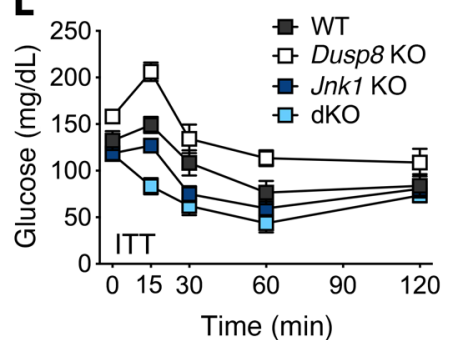

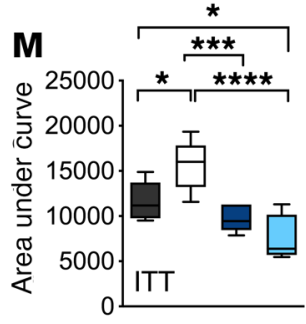

$\mathbf{N}$

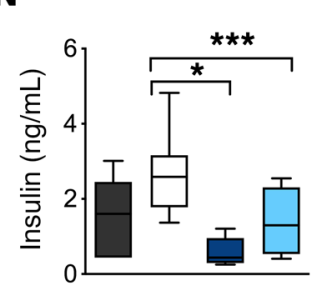

0

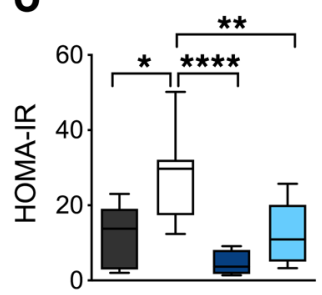

P

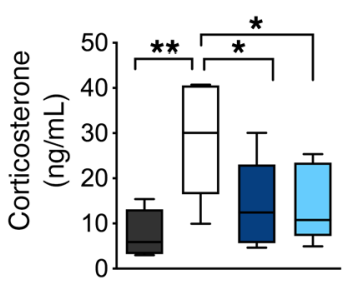


Figure 6. Glucose intolerance in HFD-fed male Dusp8-KO mice is mediated by Jnk. Representative immunohistochemical detection of c-Jun phosphorylation in hypothalamic slices of male (A) WT $(n=14)$ and (B) Dusp8-KO $(n=9)$ littermates after 18 weeks of HFD feeding and (C) counting of positively stained nuclei. (D) Pathway enrichment and (E) heatmap for MAPK signaling genes including Dusp 8 from microarray analyses of laser-capture-microdissected ARC of HFD-fed Dusp8-KO $(n=4)$ and WT $(n=3)$ mice. Relative gene expression values are shown across samples ( $z$ scales to mean expression per row). (F) Body weight and (C) body composition, (H) plasma leptin levels, and (I) markers of hypothalamic inflammation of male Dusp8-KO $(n=7)$, Jnk1-Dusp8-dKO $(n=9)$, and Jnk1-KO $(n=8)$ mice relative to WT controls $(n=6)$ were measured after 18 weeks of HFD exposure. (J-M) Glucose tolerance (GTT) and insulin tolerance tests (ITT) were carried out after 16 weeks or 17 weeks of HFD exposure, respectively ( $n=6 \mathrm{WT}, n=7$ Dusp8-KO, $n=8$ Jnk1-KO, $n=9 \mathrm{dKO}$ ). (N) Plasma insulin levels were measured in WT $(n=6)$, Dusp8-KO $(n=7)$, Jnk1-Dusp8-dKO ( $n$ $=9)$, and Ink1-KO mice $(n=8)$ after 18 weeks of HFD exposure to calculate the HOMA-IR ( $\mathbf{O})$. (P) Plasma corticosterone levels were measured in WT $(n=6)$, Dusp8-KO $(n=7)$, Jnk1-Dusp8-dKO $(n=9)$, and Jnk1-KO mice $(n=7)$ after 18 weeks of HFD. Data are shown as box-and-whisker plots (C, F-I, K, and $\mathbf{M}-\mathbf{P}$ ) or as means \pm SEM (J and $\mathbf{L})$. Scale bars: $200 \mu \mathrm{m} .{ }^{*} P<0.05$; ${ }^{* *} P$ $<0.01$; ${ }^{* *} P<0.001 ;{ }^{* * * *} P<0.0001$ by Student's $t$ test (C), 1-way ANOVA (F-I, K, and $\mathbf{M}-\mathbf{P}$ ), or 2-way ANOVA (J and $\mathbf{L}$ ).

sistent with several reports showing that decreasing Jnk activity sensitizes insulin metabolic actions $(24,25,27,28)$.

Hypothalamic insulin resistance in humans carrying the DUSP8 diabetes-risk allele. After revealing a regulatory role of Dusp8 in the control of glucose homeostasis in mice, we next aimed to translate our findings to humans by first assessing the hypothalamic DUSP8 expression in human brain tissue collected by the Netherlands Brain Bank (29). In the infundibular nucleus of T2D patients the expression of DUSP8 was significantly increased compared with nondiabetic control subjects (Figure 7A).

Next, we assessed the hypothalamic insulin sensitivity of human carriers of the minor $\mathrm{T}$ allele in the intergenic $\mathrm{T} 2 \mathrm{D}$ risk variant rs2334499. Built on our recent work that established a reduced cerebral blood flow $(\mathrm{CBF})$ after nasal insulin as a marker of high hypothalamic insulin sensitivity (30), we used MRI to quantify $\mathrm{CBF}$ in the hypothalamus (Figure 7B) before and 15 minutes after application of intranasal insulin in 47 volunteers (Supplemental Table 3). The insulin-induced change in hypothalamic blood flow was significantly associated with the DUSP8 SNP rs2334499, with a stronger insulin-dependent suppression of $\mathrm{CBF}$ in major $\mathrm{C}$ allele carriers $(\mathrm{C} / \mathrm{C})(P=0.0345$, adjusted for sex; $P=0.0334$, adjusted for sex, age, BMI) shown by the lower values of change in hypothalamic CBF levels compared with heterozygous $(\mathrm{C} / \mathrm{T})$ or minor $\mathrm{T}$ allele carriers (T/T) (Figure 7C). Stratification by sex then revealed that this association between impaired hypothalamic insulin sensitivity and the DUSP 8 variant was driven by a reduced insulininduced suppression of hypothalamic CBF levels in men (Figure 7D; $P=0.0131 ; P=0.0054$, adjusted for age, BMI) but not women (Figure 7E; $P=0.9006 ; P=0.9737$, adjusted for age, BMI) carrying the DUSP8 SNP rs2334499 minor allele (T/T).

$\mathrm{CBF}$ was further assessed in the hippocampus (Figure $7 \mathrm{~F}$ ) as a second insulin-sensitive and glucoregulatory brain region (31). We chose the hippocampus because of our recent work that revealed a smaller hippocampal size in Dusp8-KO mice, and reduced volumes of the hippocampal subregions subiculum and CA4 in humans carrying the risk variant rs2334499:C $>\mathrm{T}$ (16). We did not observe any differences in hippocampal CBF in response to intranasal insulin between the major, heterozygous or minor allele carriers regardless of their sex (Figure 7, G-I). Finally, whole-body insulin sensitivity was assessed by an oral glucose tolerance test (OGTT), but no correlation of the OGTT-derived insulin sensitivity index with the DUSP8 SNP rs2334499 in these volunteers was found ( $P$ $=0.7$, adjusted for sex; $P=0.9$, adjusted for sex, age, BMI) (Supplemental Table 3). Similarly, plasma cortisol levels were assessed but no difference between rs2334499 genotypes regardless of sex was found (Supplemental Table 3). Taken together, these findings from murine and human studies indicate that Dusp8 plays a role specifically in hypothalamic insulin sensitivity.

\section{Discussion}

We here describe a functional role for the GWAS target DUSP8 in the hypothalamic regulation of glucose homeostasis. DUSP8 was identified as a potentially novel T2D risk gene in GWAS (9) and metaanalyses coalescing GWAS cohorts (32). In a small but well-characterized cohort, we were able to show that homozygous male carriers of the minor frequency risk allele for DUSP8 have impaired hypothalamic insulin sensitivity. We further showed increased DUSP8 mRNA levels in the infundibular nucleus of individuals with T2D. Murine studies further revealed systemic glucose intolerance and insulin resistance in male Dusp8 lossof-function models exposed to HFD. Mechanistically, loss of the phosphatase Dusp8 was linked with Jnk hyperactivation in the $\mathrm{MBH}$, leading to diminished GR activity, an impairment of HPA axis feedback, and aggravation of systemic glucose tolerance due to basal hypercorticosteronemia driven by CRH neurons. Overall, our findings demonstrate a regulatory role of Dusp8 in hypothalamic inflammation, HPA axis reactivity, and insulin sensitivity.

Collectively, our experiments revealed sex-specific effects on insulin sensitivity in mice and humans that were only present in male but not female subjects. This is in line with a previous report (9) where the association of the analyzed DUSP8 SNP rs2334499 with T2D was also limited to male subjects. Reasons for the sex specificity remain elusive, but the absence of effects on glucocorticoid action or hypothalamic inflammation in female Dusp 8 -KO mice may entail direct effects of sex hormones such as estradiol (33) on HPA axis circuitry or peripheral glucoregulatory organs. Overall, our findings resonate with the emerging consensus that sex as a biological variable has to be a special focus in murine models and human studies. Another sex-related but entirely different phenomenon is the observation of the initial GWAS showing that the T2D risk associated with rs2334499 was only inherited with the paternal allele (8). Such parent-of-origin information for the alleles was unfortunately not available in our cohort of volunteers. However, not including the family data in our analyses most likely led us to underestimate the effect sizes of the detected association, as we pooled the paternally inherited allele carrying the increased diabetes risk with the maternally inherited allele. The DUSP8 SNP rs2334499 itself falls within close proximity of imprinted genes, but the region harboring it was reported to not be imprinted in human tissues or in mice, which was confirmed by clear biallelic expression for DUSP8 in humans (8). Consistent with this, we did not observe a phenotype in HFD-fed heterozygous Dusp8- 

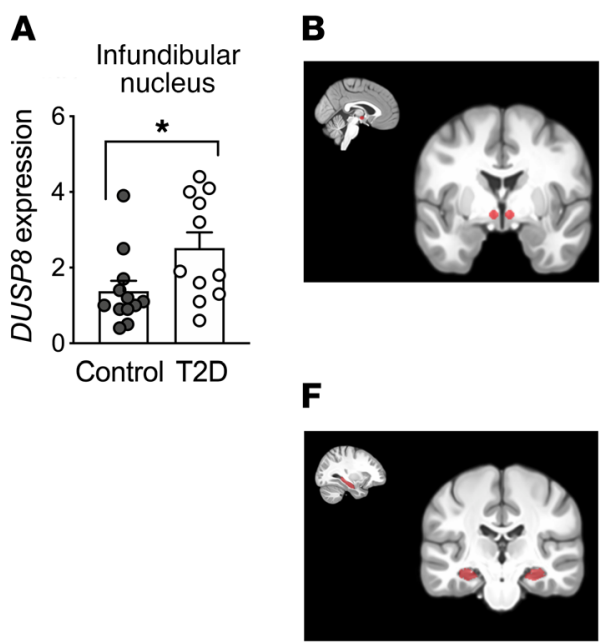

C

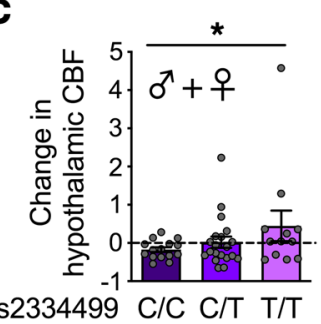

G

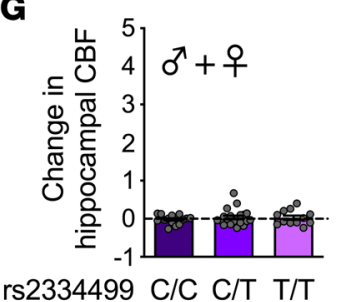

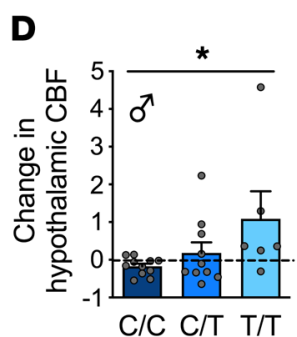

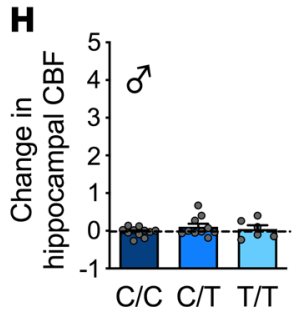

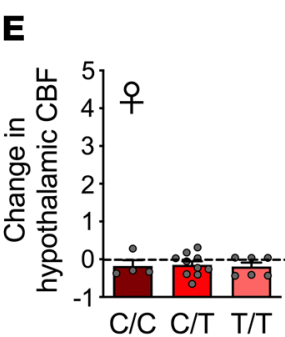

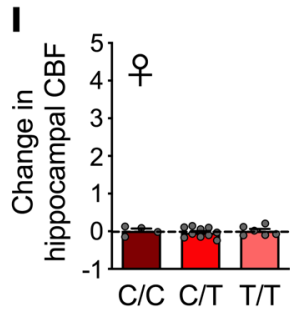

Figure 7. Increased DUSP8 expression in the infundibular nucleus of T2D patients and hypothalamic insulin resistance in humans carrying the DUSP8 diabetes-risk allele. (A) DUSP8 expression in the infundibular nucleus of humans without ( $n=12$ control) or with T2D ( $n=11)$. (B) Representative MRI brain images indicate the location of the hypothalamic region on the anatomical template in red. Intranasal insulin in healthy normal-weight individuals is known to decrease cerebral blood flow (CBF) in the hypothalamus (30). Here, we extracted the relative change in CBF (after insulin spray divided by before spray) to investigate insulin action in the hypothalamus based on the DUSP8 SNP rs2334499 genotypes. (C) The hypothalamic insulin response is displayed as the change in regional CBF after $160 \mathrm{U}$ nasal insulin. Colors represent the DUSP8 rs2334499 genotypes ( $n=15$ major allele [C/C], $n=20$ heterozygous $[C / T], n=12$ minor allele $[T / T])$ in all subjects. CBF levels after stratification for sex are displayed for (D) male $(n=11 \mathrm{C} / \mathrm{C}, n=10 \mathrm{C} / \mathrm{T}, n=6 \mathrm{~T} / \mathrm{T})$ and $(\mathrm{E})$ female ( $n=4 \mathrm{C} / \mathrm{C}, n=10 \mathrm{C} / \mathrm{T}, n=6 \mathrm{~T} / \mathrm{T}$ ) DUSP8 SNP rs2334499 carriers. (F) Representative MRI brain images with the hippocampal region displayed in red. The change in hippocampal CBF in response to intranasal insulin is displayed for (C) all subjects, (H) only men, and (I) only female participants. Data are shown as scatter dot plots. ${ }^{*} P<0.05$ by Wilcoxon's rank-sum test $(\mathbf{A})$ or multiple linear regression analysis (C-E and $\mathbf{G}-\mathbf{I}$ ).

KO males regardless of whether the mutated allele was inherited from the sire or dam.

Hypothalamic inflammation has been linked to impaired glucose metabolism and insulin sensitivity in obesity experimental models $(34,35,36)$ and in humans $(37)$. Acute hypothalamic inflammation is induced by long-chain saturated fatty acids that accumulate in the hypothalamus after crossing the blood-brain barrier (34), where they activate pathways such as IKK $\beta / \mathrm{NF}-\mathrm{\kappa B}$ (38) to ultimately enhance the expression of proinflammatory genes in the hypothalamus $(28,34)$, as observed in our Dusp8-KO model. Our finding of exacerbated hypothalamic Jnk activation, hypothalamic inflammation, and an impairment of systemic glucose homeostasis in HFD-fed Dusp8-KO mice is moreover consistent with earlier reports that linked HFD-induced activation of Jnk signaling with both hypothalamic inflammation (28) and impaired glucose metabolism (25). Our hypothesis of a Jnkdriven phenotype is further supported by a recent report on the restoration of hypothalamic insulin action after the pharmacological inhibition of Jnk (39).

Mechanistically, our data suggest that hypothalamic Jnk hyperactivation due to Dusp8 ablation causes an impairment of GR activity (22) and aberrant negative HPA axis feedback, which ultimately drives excessive glucocorticoid secretion. The latter is a well-known risk factor for T2D $(14,40)$ that perturbs hepatic gluconeogenesis and glycogen storage as well as glucose uptake in skeletal muscle and WAT (41). We observed impaired glucose homeostasis due to elevated basal corticosterone levels and aberrant hepatic expression profiles of glucoregulatory enzymes in global HFD-fed Dusp8-KO males. Moreover, we found hyperacti- vation of Jnk signaling in the ARC of both global Dusp8-KO and Dusp $8^{\text {CRH-Cre }} \mathrm{KO}$ male mice. This finding appears paradoxical, as it suggests that a Jnk-mediated impairment of the negative HPA axis feedback control in Dusp8-KO mice involves CRH neurons located (with their cell bodies) in the paraventricular nucleus (PVN), but originates in the ARC. Our data are nonetheless consistent with an earlier report that assessed the GR-mediated feedback inhibition of corticosterone on CRH release from PVN neurons (42). Specifically, when a GR antagonist was injected into the PVN where the CRH neurons were located, unperturbed feedback inhibition on adrenal corticosterone production was observed. In contrast, injection of the GR antagonist into the ARC disrupted the negative feedback, indicated by persistently increased circulating corticosterone levels (42). At present, we can only speculate on the nature of this effect. Multiple studies have established dense communication between both areas, and ARC-specific AgRP neurons were shown to require hormonal input from $\mathrm{CRH}$ neurons (via CRFR1 receptor activation) to adapt to environmental challenges (20). Ablation of Dusp8 from AgRP neurons was, however, without effect on energy and glucose metabolism. Alternatively, Dusp8-positive CRH neuronal processes located within the ARC and close to the median eminence may be susceptible to the effects of circulating corticosterone, a process perturbed locally in the ARC by hypothalamic inflammation. The phenomenon, i.e., impaired negative feedback of corticosterone on CRH release that is driven by the ARC, remains unresolved and is a limitation of our study that warrants future investigation.

Impaired HPA axis feedback inhibition in Dusp8-KO mice subjected to a Dex/CRH test, and the rescue of glucose intolerance 
in Dusp8-KO mice subjected to AAV-mediated Dusp8 overexpression in the $\mathrm{MBH}$, nonetheless corroborates an important role for hypothalamic Dusp8 as a regulator of Jnk1-driven feedback inhibition of the HPA axis. Our finding of reduced sympathetic outflow in HFD-fed Dusp8-KO mice was further in accordance with studies in humans showing glucocorticoid-induced sympathoinhibition $(12,13)$. Similarly, when patients with metabolic syndrome were stratified for insulin resistance and challenged with a glucose bolus, they showed blunted sympathetic activity and reduced NE clearance from plasma compared with insulin-sensitive patients with metabolic syndrome (43). Taken together, these data indicate that impaired glucocorticoid feedback in the hypothalamus and the reduction in sympathetic nervous system tone contribute to the insulin resistance observed in our Dusp8-KO model.

In line with the central role for Jnk1 in the regulation of glucose metabolism $(24,25)$, codeletion of Jnk1 normalized glucose tolerance and hypercorticosteronemia in HFD-fed Dusp8-KO mice. However, significant MAPK crosstalk (2) and Dusp8 phosphatase activity toward other Jnk isoforms (44) or p38 (11) have been reported. Accordingly, our data cannot fully exclude that MAPKs other than Jnk1 play a role in the etiology of the glucose intolerance phenotype of HFD-fed Dusp 8 -KO males. Similarly, our findings do not exclude a role for Dusp8 in hypothalamic subpopulations distinct from CRH neurons, in other brain areas, or in peripheral tissues. Moreover, the ablation of Dusp8 may exert distinct effects in each of these subpopulations, areas, or tissues, and future studies are thus warranted to address these current limitations. Based on our data on Dusp8-KO, p38 $8^{\mathrm{AF}}$ dominant negative (DN) double mutants, we can already exclude a potential role for p38. Similarly, our data do not point toward ERK as potential mediator of impaired glucose metabolism in HFD-fed Dusp8-KO males, as hypothalamic ERK was shown to be prominently involved in the control of food intake, BW, and thermogenic sympathetic outflow, but not in the regulation of glucose homeostasis (45). Collectively, our data support a model whereby hypothalamic Dusp8 becomes activated upon DIO to control and change the magnitude of hypothalamic Jnk activity. Dusp8 thus serves as gatekeeper against the deleterious effects that chronically increased Jnk signaling has on the regulation of energy and glucose homeostasis $(2,24)$.

Hypothalamic insulin resistance was present in HFD-fed Dusp8-KO mice and human carriers of the DUSP8 T2D risk allele. Stimulating brain insulin action by intranasal insulin improves whole-body insulin sensitivity in healthy human men, but not in obese patients with hypothalamic insulin resistance $(46,47)$. We have previously linked hypothalamic insulin responsiveness with pancreatic insulin secretion (48) and revealed an elevated second-phase insulin secretion in humans upon hypothalamic insulin action (49). Similarly, we showed that hypothalamic insulin action was linked with suppressed endogenous glucose production and elevated glucose uptake (47) as well as alterations in parasympathetic output (50). Studies in young men further linked intranasal insulin administration with attenuated HPA axis activity (51), but molecular underpinnings for this central action of insulin remained unresolved (52). Future studies are thus warranted to clarify the impact of central insulin, and rs2334499 polymorphisms, on the HPA axis of human subjects with or without T2D.
Overall, our results indicate a multisystemic process whereby hypothalamic insulin resistance, combined with deregulated HPA axis reactivity, autonomous nervous system activity, and corticosterone release, exacerbates the detrimental effects of chronic HFD feeding on systemic glucose homeostasis in male Dusp8-deficient mice via increased hypothalamic Jnk activity. Importantly, these effects appear to translate into the human situation, where individuals with $\mathrm{T} 2 \mathrm{D}$ had increased DUSP8 expression in the infundibular nucleus and male carriers of T2D risk variant rs2334499 displayed impaired hypothalamic insulin sensitivity. Replication of our human finding would be central, but our findings already extend previous GWAS that suggest Dusp 8 as a T2D risk variant. In sum, we reveal that hypothalamic Dusp8 is a crucial gatekeeper for the Jnk-dependent control of systemic glucose homeostasis.

\section{Methods}

Animals. C57BL/6J mice were obtained from Janvier and Lep $p^{o b}$ (stock 000632), Jnk1-KO (stock 004319), and p38 ${ }^{\mathrm{AF}}$ DN (stock 012736) mice were obtained from The Jackson Laboratory. Dusp8-KO mice were derived from breeding of Dusp 8 heterozygous mice (21) with a pure C57BL/6J background. Jnk1-Dusp8-dKO mice were derived from breedings of Dusp 8 heterozygous mice with Jnk1 heterozygous mice that had been crossed back to the C57BL/6J background. Dusp8-KO, $\mathrm{p} 38^{\mathrm{AF}} \mathrm{DN}$ mice were generated by breeding mice heterozygous for the DN allele of $p 38$ (p38 $8^{\mathrm{AF}}$ ) with Dusp 8 heterozygous mice.

The Dusp 8 conditional KO line was generated from the

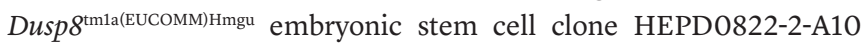
(European Mammalian Mutant Cell Repository) as detailed in the Supplemental Methods. Conditional Dusp 8 (Dusp $\left.8^{f / f f}\right)$ mice were crossed to CRH-Cre (stock 012704), AgRP-Cre (stock 012899), or POMC-Cre (stock 005965) mice (all Jackson Laboratory).

Mice at an age of 8 to 10 weeks were either maintained on chow (5.6\% fat, LM-485, Harlan Teklad or Altromin 1314) or switched to HFD (45\% kcal fat; Research Diets Inc.) for up to 30 weeks. All WT and $\mathrm{KO}$ mice used in our studies were littermates. Mice were group housed on a 12-hour light/dark cycle at $22^{\circ} \mathrm{C}$ with free access to food and water, unless indicated otherwise. Mice were distributed into experimental groups based on their BWs to assure an equal distribution of BWs at the beginning of the study. In vivo experiments were performed without blinding of the investigators. All studies were based on power analyses to assure adequate sample sizes.

Body composition analysis. Fat mass and lean mass were measured via nuclear magnetic resonance technology (EchoMRI).

GTT, ITT, and PTT. For the GTT and ITT, mice were subjected to 6 hours of fasting 1 hour after the onset of the light phase. For the PTT, mice were fasted overnight for 16 hours. Subsequently, HFD-fed mice were i.p. injected with $1.5 \mathrm{~g}$ glucose/ $\mathrm{kg}$ BW for the GTT, 0.75 or $1.0 \mathrm{U}$ insulin/kg BW (0.09 U/mL; Humalog Pen, Eli Lilly) for the ITT, and $0.75 \mathrm{~g}$ pyruvate $/ \mathrm{kg} \mathrm{BW}$ for the PTT. Chow-fed mice received $2.0 \mathrm{~g}$ glucose $/ \mathrm{kg}$ BW for the GTT and $0.75 \mathrm{U}$ insulin $/ \mathrm{kg}$ BW for the ITT. Tail blood glucose levels $(\mathrm{mg} / \mathrm{dL})$ were measured with a handheld glucometer (TheraSense Freestyle) before ( 0 minutes) and at 15, 30, 60, and 120 minutes after injection. For the glucose-stimulated insulin secretion test (GSIST), $1.5 \mathrm{mg}$ glucose/ $\mathrm{kg}$ BW was injected intravenously in 6-hour-fasted HFD-fed mice. Tail blood for insulin measurements was collected at $0,1,5,10$, and 60 minutes after injection. 
$A A V$ infusion in mice. Custom-made AAVs (serotype AAV5) with full-length murine Dusp 8 cDNA under the control of the CMV promoter or an AAV with CMV-GFP as control (pAAV5-CMV-Dusp8 vs. pAAV5CMV-EGFP; $2 \times 10^{9}$ viral genome particles $/ \mathrm{mL}$; Sirion Biotechnology) were injected bilaterally into the MBH of single-housed, DIO WT and Dusp8-KO littermates (6 months old) using a motorized stereotaxic system from Neurostar as detailed in the Supplemental Methods.

Acute insulin challenge. To assess biochemical responses to insulin stimulation, insulin was i.p. administered at a dose of $3 \mathrm{U} / \mathrm{kg} \mathrm{BW}$ in age-matched DIO WT and Dusp8-KO littermates. Control mice received vehicle instead. Mice were sacrificed 8 minutes after insulin administration by cervical dislocation. Hypothalami were collected to measure signal transduction markers by Western blotting.

Catecholamine turnover rate determination. Catecholamine turnover was measured on the basis of the decline in tissue NE content after the inhibition of catecholamine biosynthesis with $\alpha$-methyl-DL-tyrosine methyl ester hydrochloride ( $\alpha$-MPT, M3281, MilliporeSigma) as described previously (53) and in the Supplemental Methods.

Dex/CRH test. In the combined Dex/CRH test (17), the corticosterone secretion of Dusp8-KO and WT mice was monitored in response to a pharmacological suppression of adrenocortical activity with dexamethasone $(0.05 \mathrm{mg} / \mathrm{kg} \mathrm{BW})$ and a subsequent stimulation with $\mathrm{CRH}$ $(0.15 \mathrm{mg} / \mathrm{kg} \mathrm{BW})$ as detailed in the Supplemental Methods.

Metyrapone injections. Age-matched DIO WT and Dusp8-KO littermates received daily i.p. injections of $100 \mathrm{mg} / \mathrm{kg}$ BW metyrapone (catalog M2696, MilliporeSigma) for 14 days that were shown to reduce endogenous corticosterone production (18).

Immunohistochemistry and immunofluorescence. Paraformaldehyde-fixed brains were processed for cryosectioning followed by immunohistochemical and immunofluorescent staining in the free-floating approach as detailed in the Supplemental Methods.

In situ hybridization. Coronal hypothalamic sections $(16 \mu \mathrm{m})$ were cut on a cryostat and immediately stored at $-80^{\circ} \mathrm{C}$ until hybridization. Sense and antisense probes were generated from the Dusp 8 cDNA sequence using a digoxigenin (DIG) RNA labeling kit (Roche). The hybridization procedure is described in detail in the Supplemental Methods.

Blood chemistry. Blood was collected in EDTA-containing centrifuge tubes and centrifuged at $4^{\circ} \mathrm{C}$ and $2000 \mathrm{~g}$ for 10 minutes. Plasma triglycerides, cholesterol, and nonesterified fatty acids were measured by commercial enzymatic assay kits (Wako Chemicals). Insulin and leptin were measured by ultrasensitive murine insulin and murine leptin ELISA kits (Merck Millipore). Plasma corticosterone levels were measured by radioimmunoassay (MP Biomedicals; sensitivity 6.25 $\mathrm{ng} / \mathrm{mL}$ ) or corticosterone ELISA kit (Arbor Assays). All assays were performed according to the manufacturers' instructions.

Hepatic triglyceride and glycogen content measurements. Hepatic triglyceride content was determined by using a triglyceride assay kit (Wako Chemicals, 290-63701), and hepatic glycogen content was measured by the enzymatic starch analysis assay (R-Biopharm AG, 10 207748 035), according to the manufacturers' instructions.

Cell culture. HEK293 cells (Leibniz Institute DSMZ, ACC 305) were grown in Dulbecco's modified Eagle medium (DMEM) supplemented with $10 \%$ fetal calf serum and $1 \%$ penicillin/streptomycin. Cell lines were tested for mycoplasma contamination after thawing and every other month in culture. Transient transfections were performed using FuGene reagent from Promega and plasmids pCMV6 (PS100020, OriGene Technologies) or pCMV6-
Dusp8 (MR209863, OriGene) and pcDNA3.1-hGR (54) as indicated in serum-free medium. Stimulation experiments were performed 48 hours after transfection.

GR luciferase assay. For luciferase assays, HEK293 cells were seeded into $96-$-well plates and grown overnight to $70 \%-80 \%$ confluence. Cells were then transiently transfected with pGL4-Per1 (54), pRL-TK (E2241, Promega), and pCMV6 or pCMV6-Dusp8 plasmids. Twentyfour hours after transfection, cells where indicated were pretreated with anisomycin $(100 \mathrm{nM})$ overnight and then stimulated with Dex $(100 \mathrm{nM})$ for 5 hours. All samples were run in triplicate. Luciferase activity was measured using a microplate luminometer (PHERAstar, BMG Labtech) and the Dual-Glo Luciferase Assay System (Promega), according to the instructions from the manufacturers.

Protein extraction and Western blotting analysis. HEK293 cells and murine tissues were processed for protein extraction, concentration determination, and Western blot analysis as detailed in the Supplemental Methods.

Microarray analysis of laser-capture-microdissected ARC. Perfused and sucrose-equilibrated brains of global Dusp8-WT and -KO males were cut coronally followed by laser capture microdissection of the ARC. Subsequently, RNA was isolated from the ARC and gene expression was analyzed by microarray. Details are given in the Supplemental Methods.

RNA isolation and qPCR analysis. RNA was isolated from flashfrozen murine tissues and postmortem formalin-fixed, paraffinembedded (FFPE) human brain tissue with commercially available kits and reverse transcribed into cDNA followed by gene expression analysis as detailed in the Supplemental Methods.

Human data. DUSP8 expression in the infundibular nucleus was assessed in postmortem hypothalamic tissues of $11 \mathrm{~T} 2 \mathrm{D}$ and 12 nonT2D control subjects obtained from the Netherlands Brain Bank (29). Regional brain insulin sensitivity was assessed in a group of 47 healthy participants of the ongoing Tübingen Family (TUF) study (ref. 55 and Supplemental Table 3), using an established protocol that combines the measurement of CBF by functional MRI with the delivery of 160 $\mathrm{U}$ of insulin as nasal spray as described previously (30). Blood flow for the hypothalamic and hippocampal region of interest was extracted and associations with the DUSP8 SNP rs2334499 (major allele [C/C], heterozygous $[\mathrm{C} / \mathrm{T}]$, minor allele $[\mathrm{T} / \mathrm{T}]$ ) were tested by multiple linear regression analyses with adjustment for age.

Data availability. The microarray data have been submitted to the Gene Expression Omnibus (GEO) database at the NCBI (GSE112688: https://www.ncbi.nlm.nih.gov/geo/query/acc.cgi?acc=GSE112688). The mass spectrometry proteomics data have been deposited to the ProteomeXchange Consortium via the PRIDE (56) partner repository with the data set identifier PXD019451. All other data generated or analyzed during this study are available within the paper and its supplementary information file. Antibodies for the detection of proteins are given in Supplemental Table 4, and Taqman probes and primer sequences for gene expression analyses are given in Supplemental Tables 5 and 6. Last, we provide images of uncut gels for all Western blot data in the Supplemental Materials.

Limitations of human data. A replication of our human finding of reduced CBF in rs2334499 minor allele carriers would be central. As the effect size for the association with hypothalamic insulin sensitivity is much larger than the effect size of the association with diabetes risk, a considerably smaller cohort than the initial discovery cohorts for dia- 
betes risk would be sufficient for such a replication approach. Unfortunately, we are not aware of any other human cohort with quantification of hypothalamic insulin sensitivity and genetic information. Future replication is nonetheless warranted to substantiate our findings.

Statistics. In vivo measurements were taken from distinct samples and in vitro measurements were performed in at least 3 independent biological replicates. For animal experiments, we performed power analysis with the assumption of normality. Statistically significant outliers were identified by the Grubbs test. All statistical analyses (except microarray analysis) were performed using GraphPad Prism, SPSS, SAS, or JMP. Two groups were compared by using 2-tailed unpaired Student's $t$ test or the nonparametric Wilcoxon rank-sum test. Multiple comparison analyses were performed by using 1- or 2-way ANOVA followed by Tukey's or Bonferroni's post hoc tests. Combined indirect calorimetry measurements were assessed by ANCOVA, using BW as covariate. $P$ values lower than 0.05 were considered significant. Results are presented as scatter dot plots, as means \pm SEM, or as box-and-whisker-plots (median, 25th and 75th quartile; whiskers 5th-95th percentiles).

Study approval. All animal studies were approved by the State of Bavaria, Germany, or by the Institutional Animal Care and Use Committee of the University of Cincinnati. Autopsies to obtain postmortem hypothalamic tissue samples were approved by the Medical Ethic Committee of the VU Medical Center, The Netherlands. All human subjects of the TUF study provided informed written consent before the inclusion in the study and the local ethics committee of the University of Tübingen approved the protocol.

\section{Author contributions}

SCS, DGK, KP, PB, EVB, LH, FG, RJ, JC, SM, MW, TDM, KS, WW, $\mathrm{RN}, \mathrm{JDM}, \mathrm{SL}$, and PTP performed in vivo experiments in mice. JDM provided the global Dusp8-KO model. SCS, DGK, and PTP designed, performed, and analyzed the AAV infusion study. JC, SM, SL, MW, SCS, and JR planned, performed, and analyzed the in vivo delineation of insulin resistance. SCS, SL, JN, and MDA designed, performed, and analyzed the $\alpha$-MPT study. MVS, PTP, and SCS designed, performed, and analyzed the dexamethasone suppression test and the metyrapone study. FS and NK performed the phospho-proteome analysis. MI, MHA, and JB performed and analyzed the microarray study. SCS, DGK, and CXY conducted immunohistochemical staining. RN performed in situ hybridizations. SCS, EVB, and HS performed qPCRs and Western blot analyses. SCS, FCS, and CXY designed, performed, and analyzed the gene expression in postmortem human brain tissue. SK, HUH, and $\mathrm{MH}$ designed, performed, and analyzed the fMRI study and plasma measurements of humans from the TUF cohort. SCS, MVS, SL, MH, MHT, and PTP designed experiments and analyzed and interpreted the results. SCS, MH, MVS, SL, MHT, and PTP prepared the manuscript. SCS, MHT, and PTP developed the conceptual framework of this study.

\section{Acknowledgments}

We thank Sarah Jelinek, Marlene Kilian, Verónica Casquero, Anke Bettenbrock, Emilija Malogajski, Daniel Brandt, and Bea Deiml for their skillful technical assistance. We are most grateful to Aurélie Joly Amado for excellent advice on the norephinephrine turnover studies. Andreas Peter kindly performed the genotyping of the TUF cohort. Henriette Uhlenhaut kindly provided the hGR plasmid and the pGL4-Per1 luciferase assay plasmid. This work was supported in part by the Helmholtz Portfolio Program "Metabolic Dysfunction" (to MHT), by the Alexander von Humboldt Foundation (to MHT), by the Helmholtz Alliance ICEMED-Imaging and Curing Environmental Metabolic Diseases (to SCS, MHT, and HUH), by the German Center for Diabetes Research (DZD; to SCS, PP, MH, and MHA), by the Helmholtz-Israel Cooperation in Personalized Medicine (to PTP), by the Helmholtz Initiative for Personalized Medicine (iMed; to MHT), by the Helmholtz Alliance "Aging and Metabolic Programming, AMPro" (to JB and NK), by the Emmy-Noether DFG "KR5166/1-1" (to NK), by German Federal Ministry of Education and Research "01KX1012" (to MHA), and through the Initiative and Networking Fund of the Helmholtz Association.

Address correspondence to: Paul T. Pfluger, Research Unit NeuroBiology of Diabetes, Helmholtz Zentrum München $\mathrm{GmbH}$, Ingolstädter Landstraße 1, 85764 Neuherberg, Germany. Phone: 49.89.3187.2104; Email: paul.pfluger@helmholtz-muenchen.de.
1. Hotamisligil GS, Shargill NS, Spiegelman BM. Adipose expression of tumor necrosis factoralpha: direct role in obesity-linked insulin resistance. Science. 1993;259(5091):87-91.

2. Kyriakis JM, Avruch J. Mammalian MAPK signal transduction pathways activated by stress and inflammation: a 10-year update. Physiol Rev. 2012;92(2):689-737.

3. Kondoh K, Nishida E. Regulation of MAP kinases by MAP kinase phosphatases. Biochim Biophys Acta. 2007;1773(8):1227-1237.

4. Seternes OM, Kidger AM, Keyse SM. Dual-specificity MAP kinase phosphatases in health and disease. Biochim Biophys Acta Mol Cell Res. 2019;1866(1):124-143.

5. Farooq A, Zhou MM. Structure and regulation of MAPK phosphatases. Cell Signal. 2004;16(7):769-779.

6. Choi HR, et al. Dual-specificity phosphatase 10 controls brown adipocyte differentiation by modulating the phosphorylation of $\mathrm{p} 38$ mitogen-activated protein kinase. PLoS One. 2013;8(8):e72340.

7. Ferguson BS, Nam H, Morrison RF. Dual-specificity phosphatases regulate mitogen-activated protein kinase signaling in adipocytes in response to inflammatory stress. Cell Signal. 2019;53:234-245.

8. Kong A, et al. Parental origin of sequence variants associated with complex diseases. Nature. 2009;462(7275):868-874.

9. Morris AP, et al. Large-scale association analysis provides insights into the genetic architecture and pathophysiology of type 2 diabetes. Nat Genet. 2012;44(9):981-990.

10. Martell KJ, Seasholtz AF, Kwak SP, Clemens KK, Dixon JE. hVH-5: a protein tyrosine phosphatase abundant in brain that inactivates mitogen-activated protein kinase. J Neurochem. 1995;65(4):1823-1833.

11. Muda M, et al. The dual specificity phosphatases
M3/6 and MKP-3 are highly selective for inactivation of distinct mitogen-activated protein kinases. J Biol Chem. 1996;271(44):27205-27208.

12. Golczynska A, Lenders JW, Goldstein DS. Glucocorticoid-induced sympathoinhibition in humans. Clin Pharmacol Ther. 1995;58(1):90-98.

13. Cameron OG, Starkman MN, Schteingart DE. The effect of elevated systemic cortisol levels on plasma catecholamines in Cushing's syndrome patients with and without depressed mood. JPsychiatr Res. 1995;29(5):347-360.

14. Tomlinson JW, Finney J, Gay C, Hughes BA, Hughes SV, Stewart PM. Impaired glucose tolerance and insulin resistance are associated with increased adipose 11beta-hydroxysteroid dehydrogenase type 1 expression and elevated hepatic 5alpha-reductase activity. Diabetes. 2008;57(10):2652-2660.

15. Joly-Amado A, et al. Hypothalamic AgRP-neurons control peripheral substrate utilization and nutrient partitioning. EMBO J. 2012;31(22):4276-4288. 
16. Baumann P, et al. Dusp8 affects hippocampal size and behavior in mice and humans. Sci Rep. 2019;9(1):19483.

17. Touma C, et al. FK506 binding protein 5 shapes stress responsiveness: modulation of neuroendocrine reactivity and coping behavior. Biol Psychiatry. 2011;70(10):928-936.

18. Takeshita N, Yoshino T, Mutoh S. Possible involvement of corticosterone in bone loss of genetically diabetic $\mathrm{db} / \mathrm{db}$ mice. Horm Metab Res. 2000;32(4):147-151.

19. Belgardt BF, Okamura T, Bruning JC. Hormone and glucose signalling in POMC and AgRP neurons. J Physiol. 2009;587(pt 22):5305-5314.

20. Kuperman Y, et al. CRFR1 in AgRP neurons modulates sympathetic nervous system activity to adapt to cold stress and fasting. Cell Metab. 2016;23(6):1185-1199.

21. Liu R, van Berlo JH, York AJ, Vagnozzi RJ, Maillet $\mathrm{M}$, Molkentin JD. DUSP8 regulates cardiac ventricular remodeling by altering ERK1/2 signaling. Circ Res. 2016;119(2):249-260.

22. Rogatsky I, Logan SK, Garabedian MJ. Antagonism of glucocorticoid receptor transcriptional activation by the c-Jun N-terminal kinase. Proc Natl Acad Sci U S A. 1998;95(5):2050-2055.

23. Campbell JN, et al. A molecular census of arcuate hypothalamus and median eminence cell types. Nat Neurosci. 2017;20(3):484-496.

24. Hirosumi J, et al. A central role for JNK in obesity and insulin resistance. Nature. 2002;420(6913):333-336.

25. Belgardt BF, et al. Hypothalamic and pituitary c-Jun N-terminal kinase 1 signaling coordinately regulates glucose metabolism. Proc Natl Acad Sci U S A. 2010;107(13):6028-6033.

26. Wong ES, et al. p38MAPK controls expression of multiple cell cycle inhibitors and islet proliferation with advancing age. Dev Cell. 2009;17(1):142-149.

27. Aguirre V, Uchida T, Yenush L, Davis R, White MF. The c-Jun NH(2)-terminal kinase promotes insulin resistance during association with insulin receptor substrate-1 and phosphorylation of Ser(307). J Biol Chem. 2000;275(12):9047-9054.

28. De Souza CT, et al. Consumption of a fat-rich diet activates a proinflammatory response and induces insulin resistance in the hypothalamus. Endocrinology. 2005;146(10):4192-4199.

29. Hogenboom R, et al. Loss of arginine vasopressin- and vasoactive intestinal polypeptide-containing neurons and glial cells in the suprachiasmatic nucleus of individuals with type 2 diabetes. Diabetologia. 2019;62(11):2088-2093.
30. Kullmann S, et al. Selective insulin resistance in homeostatic and cognitive control brain areas in overweight and obese adults. Diabetes Care. 2015;38(6):1044-1050.

31. Soto M, Cai W, Konishi M, Kahn CR. Insulin signaling in the hippocampus and amygdala regulates metabolism and neurobehavior. Proc Natl Acad Sci U S A. 2019;116(13):6379-6384.

32. Billings LK, Florez JC. The genetics of type 2 diabetes: what have we learned from GWAS? Ann N Y Acad Sci. 2010;1212:59-77.

33. Hong J, Stubbins RE, Smith RR, Harvey AE, Nunez NP. Differential susceptibility to obesity between male, female and ovariectomized female mice. Nutr J. 2009;8:11.

34. Posey KA, et al. Hypothalamic proinflammatory lipid accumulation, inflammation, and insulin resistance in rats fed a high-fat diet. Am J Physiol Endocrinol Metab. 2009;296(5):E1003-12.

35. Jais A, Bruning JC. Hypothalamic inflammation in obesity and metabolic disease. J Clin Invest. 2017;127(1):24-32.

36. Romanatto T, et al. TNF-alpha acts in the hypothalamus inhibiting food intake and increasing the respiratory quotient--effects on leptin and insulin signaling pathways. Peptides. 2007;28(5):1050-1058.

37. Kreutzer C, et al. Hypothalamic inflammation in human obesity is mediated by environmental and genetic factors. Diabetes. 2017;66(9):2407-2415.

38. Kleinridders A, et al. MyD88 signaling in the CNS is required for development of fatty acid-induced leptin resistance and diet-induced obesity. Cell Metab. 2009;10(4):249-259.

39. Benzler J, et al. Acute inhibition of central c-Jun $\mathrm{N}$-terminal kinase restores hypothalamic insulin signalling and alleviates glucose intolerance in diabetic mice. J Neuroendocrinol. 2013;25(5):446-454.

40. Di Dalmazi G, Pagotto U, Pasquali R, Vicennati V. Glucocorticoids and type 2 diabetes: from physiology to pathology. J Nutr Metab. 2012;2012:525093.

41. de Guia RM, Rose AJ, Herzig S. Glucocorticoid hormones and energy homeostasis. Horm Mol Biol Clin Investig. 2014;19(2):117-128.

42. Leon-Mercado L, Herrera Moro Chao D, Basualdo MD, Kawata M, Escobar C, Buijs RM. The arcuate nucleus: a site of fast negative feedback for corticosterone secretion in male rats. eNeuro. 2017;4(1):e0350-16.2017.

43. Straznicky NE, et al. Blunted sympathetic neural response to oral glucose in obese subjects with the insulin-resistant metabolic syndrome. Am J Clin Nutr. 2009;89(1):27-36.
44. Oehrl W, Cotsiki M, Panayotou G. Differential regulation of M3/6 (DUSP8) signaling complexes in response to arsenite-induced oxidative stress. Cell Signal. 2013;25(2):429-438.

45. Rahmouni K, Sigmund CD, Haynes WG, Mark AL. Hypothalamic ERK mediates the anorectic and thermogenic sympathetic effects of leptin. Diabetes. 2009;58(3):536-542.

46. Heni M, Kullmann S, Preissl H, Fritsche A, Haring HU. Impaired insulin action in the human brain: causes and metabolic consequences. Nat Rev Endocrinol. 2015;11(12):701-711.

47. Heni M, et al. Hypothalamic and striatal insulin action suppresses endogenous glucose production and may stimulate glucose uptake during hyperinsulinemia in lean but not in overweight men. Diabetes. 2017;66(7):1797-1806.

48. Kullmann S, et al. Hypothalamic insulin responsiveness is associated with pancreatic insulin secretion in humans. Physiol Behav. 2017;176:134-138.

49. Heni M, et al. Insulin action in the hypothalamus increases second phase insulin secretion in humans [published online November 5, 2019]. Neuroendocrinology. https://doi. org/10.1159/000504551.

50. Heni M, et al. Central insulin administration improves whole-body insulin sensitivity via hypothalamus and parasympathetic outputs in men. Diabetes. 2014;63(12):4083-4088.

51. Benedict $\mathrm{C}$, et al. Intranasal insulin improves memory in humans. Psychoneuroendocrinology. 2004;29(10):1326-1334.

52. Plum L, Schubert M, Bruning JC. The role of insulin receptor signaling in the brain. Trends Endocrinol Metab. 2005;16(2):59-65.

53. Brodie BB, Costa E, Dlabac A, Neff NH, Smookler HH. Application of steady state kinetics to the estimation of synthesis rate and turnover time of tissue catecholamines. JPharmacol Exp Ther. 1966;154(3):493-498.

54. Uhlenhaut $\mathrm{NH}$, et al. Insights into negative regulation by the glucocorticoid receptor from genome-wide profiling of inflammatory cistromes. Mol Cell. 2013;49(1):158-171.

55. Stumvoll M, et al. Association of the T-G polymorphism in adiponectin (exon 2) with obesity and insulin sensitivity: interaction with family history of type 2 diabetes. Diabetes. 2002;51(1):37-41.

56. Perez-Riverol Y, et al. The PRIDE database and related tools and resources in 2019: improving support for quantification data. Nucleic Acids Res. 2019;47(D1):D442-D50. 